\title{
Rotational Symmetries and Spherical Measure in Homogeneous Groups
}

\section{Valentino Magnani ${ }^{1}$}

Received: 3 November 2020 / Accepted: 13 January 2022 / Published online: 1 February 2022

(c) The Author(s) 2022

\section{Abstract}

We present different types of rotational symmetries for distances in homogeneous groups, showing that the area formula for the associated spherical measure takes a simple form.

Keywords Homogeneous group · Area formula $\cdot$ Spherical measure $\cdot$ Centered Hausdorff measure $\cdot$ Homogeneous distance $\cdot$ Rotational symmetry

Mathematics Subject Classification $28 \mathrm{~A} 75 \cdot 58 \mathrm{C} 35 \cdot 43 \mathrm{~A} 80 \cdot 22 \mathrm{E} 25$

\section{Contents}

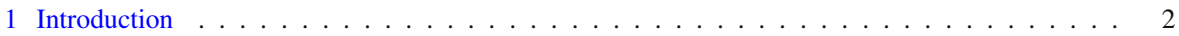

2 Basic Notions . . . . . . . . . . . . . . . . . . . . . . . 6

3 Vertically Symmetric Distances . . . . . . . . . . . . . . . . . . . . . . 8

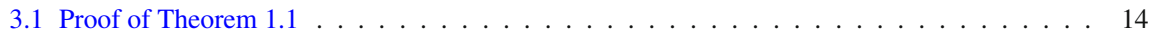

4 Area and Coarea Formulas in Low Codimension . . . . . . . . . . . . . . . . . . . . . . . 17

5 Multiradial Distances . . . . . . . . . . . . . . . . . . . . . . . . 20

5.1 Multiradial Distances in Step Two Homogeneous Groups . . . . . . . . . . . . . . . . . . 21

5.2 One Dimensional Rotational Symmetries . . . . . . . . . . . . . . . . . . . . . 24

5.3 Proof of Theorem 1.3 and Applications . . . . . . . . . . . . . . . . . . . 25

6 Sections of Convex Balls . . . . . . . . . . . . . . . . . . . . . . . . . . . . . . 27

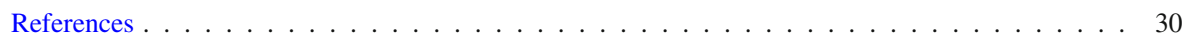

Dedicated to Dr. Piero Vincenzo Lippolis, with deep esteem.

The author acknowledges the support of the University of Pisa, Project PRA 201849.

Valentino Magnani

valentino.magnani@unipi.it

1 Dipartimento di Matematica, Università di Pisa, Largo Bruno Pontecorvo 5, 56127 Pisa, Italy 


\section{Introduction}

The problem of computing the spherical measure of submanifolds in homogeneous groups still has a number of questions to be investigated. Its intriguing aspect is how it naturally involves the algebraic structure of the group and the "tangential properties" of the submanifold. The anisotropic infinitesimal behavior of the submanifold in many cases leads to new geometric questions.

Our motivations have their roots in the wider project to expand results and tools of Geometric Measure Theory in the framework of noncommutative homogeneous groups. A fundamental concept is that of area, that is obtained using the spherical measure with respect to the distance of the group. To our knowledge, the first integral formulas for this measure date back to the work of Pansu [29] in Heisenberg groups, and of Heinonen [14] in Carnot groups. Related developments appeared later in connection with the study of BV functions, sets of finite perimeter, intrinsic rectifiable sets, currents and characterizations of intrinsic regular submanifolds. Due to the large and always expanding literature, we limit ourselves to mention some related works, $[1,2,4,5,9-12,15,21,22,27,28,31]$ and the references therein, warning the reader that this list is very far from being complete.

In the present work, we focus our attention on the role of symmetries of distances in computing the spherical measure of $C^{1}$ smooth submanifolds. Our results constitute a continuation of those in [23], that together with this paper arise from the former preprint [24]. However our tools also apply to intrinsic regular sets, as for instance in [5] and [31]. Indeed, intrinsic regular sets might be very far from being smooth in the standard sense, [16]. For such reason, area formulas in noncommutative homogenous groups cannot rely on classical results of Geometric Measure Theory and represent a rather challenging question.

Let $\Sigma$ be an n-dimensional $C^{1}$ smooth submanifold of degree $\mathrm{N}$ in a homogeneous group $\mathbb{G}$ equipped with a homogeneous distance $d$. The analytic notions of degree $d(\Sigma)$ of a submanifold $\Sigma$ and of pointwise degree $d_{\Sigma}(p)$ at $p \in \Sigma$ were introduced in [26]. We will refer to formulas (2.10) and (2.11) of [23, Sect. 2.3]. For each Borel set $B \subset \Sigma$, the area formula reads as follows

$$
\int_{B}\left\|\tau_{\Sigma, \mathrm{N}}^{\tilde{g}}(p)\right\| d \operatorname{vol}_{\tilde{g}}(p)=\int_{B} \beta_{d}\left(A_{p} \Sigma\right) d \mathcal{S}^{\mathrm{N}}(p) .
$$

The integral on the left hand side represents the intrinsic measure of $\Sigma$, introduced in [26], see for instance [23, Definition 7.3]. The spherical measure $\mathcal{S}^{\mathrm{N}}$ is constructed by the distance $d$. We have denoted by $\beta_{d}\left(A_{p} \Sigma\right)$ the spherical factor with respect to $A_{p} \Sigma$ (Definition 1.1). The homogeneous tangent space $A_{p} \Sigma$ at $p$ has been introduced in [23, Definition 2.7]. From a geometric viewpoint, the spherical factor $\beta_{d}(S)$ represents the maximal area among all intersections of $S$ with metric unit balls, that stay at a bounded distance from the origin.

Definition 1.1 (Spherical factor) Let $S \subset \mathbb{G}$ be a linear subspace of dimension $\mathrm{n}$ and consider a homogeneous distance $d$ on $\mathbb{G}$. Let $|\cdot|$ denote the norm arising from the fixed graded scalar product on $\mathbb{G}$. The spherical factor of $d$ with respect to $S$ is the number 


$$
\beta_{d}(S)=\max _{d(u, 0) \leq 1} \mathcal{H}_{|\cdot|}^{\mathrm{n}}(\mathbb{B}(u, 1) \cap S)
$$

where $\mathbb{B}(u, 1)=\{v \in \mathbb{G}: d(v, u) \leq 1\}$ and $\mathcal{H}_{|\cdot|}^{\mathrm{n}}$ is defined in (2.7).

Our main interest is in finding those conditions on the homogeneous distance $d$ such that $\beta_{d}(S)$ is constant as $S \subset \mathbb{G}$ varies in a specific class of subspaces. In the applications such subspaces will be the homogeneous tangent spaces of a submanifold.

The next definition will state the constancy of $\beta_{d}$ with respect to a fixed family $\mathcal{F}$ of homogeneous subspaces. As in the previous definition, we refer to a fixed graded scalar product on $\mathbb{G}$ and use the notion of homogeneous subspace (Sect. 2).

Definition 1.2 (Rotationally symmetric distance) Let $d$ be a homogeneous distance on a homogeneous group $\mathbb{G}$ and let $\mathcal{F}$ be a nonempty family of homogeneous subspaces of $\mathbb{G}$. We say that $d$ is rotationally symmetric with respect to $\mathcal{F}$ if the spherical factor $\beta_{d}$ is a constant function on $\mathcal{F}$. We denote this constant by $\omega_{d}(\mathcal{F})$.

If all homogeneous tangent spaces of a submanifold $\Sigma$ are contained in a family $\mathcal{F}$ and $d$ is rotationally symmetric with respect to $\mathcal{F}$, then (1.1) immediately yields a neat formula for the integral representation of the spherical measure. Denoting by $\omega_{d}(\mathcal{F})$ the constant spherical factor and setting $\mathcal{S}_{d}^{\mathrm{N}}=\omega_{d}(\mathcal{F}) \mathcal{S}^{\mathrm{N}}$, where $\mathcal{S}^{\mathrm{N}}$ is introduced in (2.6), then (1.1) yields

$$
\mathcal{S}_{d}^{\mathrm{N}}(B)=\int_{B}\left\|\tau_{\Sigma, \mathrm{N}}^{\tilde{g}}(p)\right\| d \operatorname{vol}_{\tilde{g}}(p),
$$

that has the form of the standard area formula for Riemannian manifolds, [7, 3.2.46]. The simplest example of constant spherical factor is in the Euclidean space, that can be seen as a commutative homogeneous group $\mathbb{G} \approx \mathbb{R}^{\mathrm{q}}$ of step one, equipped with the Euclidean distance $d_{E}$. The spherical factor of an n-dimensional submanifold $\Sigma$ becomes

$$
\beta_{d_{E}}\left(A_{p} \Sigma\right)=\omega_{d_{E}}\left(\mathcal{F}_{\mathrm{q}, \mathrm{n}}\right)
$$

for all $p \in \Sigma$ and the homogeneous tangent space $A_{p} \Sigma$ coincides with the standard tangent space. The constant $\omega_{d_{E}}\left(\mathcal{F}_{\mathrm{q}, \mathrm{n}}\right)$ is the Lebesgue measure of the unit ball in $\mathbb{R}^{\mathrm{n}}$. We have denoted by $\mathcal{F}_{\mathrm{q}, \mathrm{n}}$ the Grassmannian of n-dimensional subspaces of $\mathbb{G} \approx \mathbb{R}^{\mathrm{q}}$. As soon as we consider any commutative homogeneous group of step one, then the spherical factor need not be constant. We are actually dealing with a finite dimensional Banach space, where a suitable area formula for submanifolds was proved in [3, Theorem 4.2].

The general question about the constancy of $\beta_{d}$ is nontrivial, since it depends on the algebraic and metric structure of the group. A general statement about symmetries in homogeneous groups would involve infinitely many homogeneous groups, that are not isomorphic to each other, hence their distances are not bi-Lipschitz equivalent. The fact that we have such infinitely many "different geometries" makes general symmetry results rather challenging. 
Our first case concerns rotationally symmetric distances with respect to the family $\mathcal{N}_{\mathrm{n}}$ of all n-dimensional vertical subgroups (Definition 3.1). Vertical subgroups are the homogeneous tangent spaces at some points of transversal submanifolds, [23]. As a result, such rotationally symmetric distances imply a simpler integral form for the spherical measure of transversal submaniofolds, according to Theorem 1.2 below. The family of transversal manifolds includes all hypersurfaces and the larger class of non-horizontal submanifolds, [19]. For instance, in Heisenberg groups a smooth submanifold is either Legendrian or transversal.

The rotational symmetry with respect to $\mathcal{N}_{\mathrm{n}}$ is a property of n-vertically symmetric distances (Definition 3.2) that is studied in Sect. 3. An interesting aspect of such distances is their symmetry with respect to special classes of isometries, that preserve the shape of the metric unit ball, although they are not necessarily Lie group homomorphisms. The informal justification for this resides in the blow-up process, that suggests us to look at the metric unit ball as embedded in the tangent space, where we only have a linear structure. Vertical symmetric distances somehow possess the "minimal rotational symmetries" such that the following theorem holds.

Theorem 1.1 If $d$ is an $\mathrm{n}$-vertically symmetric distance, then $d$ is rotationally symmetric with respect to the family $\mathcal{N}_{\mathrm{n}}$ of all $\mathrm{n}$-dimensional vertical subgroups.

Sections 3 and 5 provide different examples of n-vertically symmetric distances. Theorem 1.1 has been used in [5] to establish a simpler form of the area formula of low codimensional $\mathbb{H}$-regular surfaces. Combining Theorem 1.1 and the area formula established in $[23,(1.7)]$, we arrive at the following consequence.

Theorem 1.2 Let d be an n-vertically symmetric distance on the homogeneous group $\mathbb{G}$ and denote by $\omega_{d}\left(\mathcal{N}_{\mathrm{n}}\right)$ its constant spherical factor. We define the spherical measure $\mathcal{S}_{d}^{\mathrm{Q}_{\mathrm{n}}}=\omega_{d}\left(\mathcal{N}_{\mathrm{n}}\right) \mathcal{S}^{\mathrm{Q}_{\mathrm{n}}}$, where $\mathrm{Q}_{\mathrm{n}}$ is as in (3.4). If $\Sigma \subset \mathbb{G}$ is an $\mathrm{n}$-dimensional transversal submanifold, then for any Borel set $B \subset \Sigma$ we have

$$
\mathcal{S}_{d}^{\mathrm{Q}_{\mathrm{n}}}\left\llcorner\Sigma(B)=\int_{B}\left\|\tau_{\Sigma, \mathrm{Q}_{\mathrm{n}}}^{\tilde{g}}(p)\right\|_{g} d \sigma_{\tilde{g}}(p) .\right.
$$

A byproduct of Theorem 1.2 is a simple form of area and coarea formulas in low codimension. Let $\mathrm{Q}$ denote the Hausdorff dimension of $\mathbb{G}$. If the codimension $k$ of a submanifold is less than the dimension $m$ of the first layer of $\mathbb{G}$, then the submanifold is transversal if and only if it is non-horizontal and if and only if its degree is $\mathrm{Q}-k$. So the area formula (1.3) for n-vertically symmetric distances also applies to non-horizontal submanifolds, giving simpler formulas, like (4.2), (4.3) and (4.4). As a consequence, a standard form of the coarea formula for $\mathbb{R}^{k}$-valued Riemannian Lipschitz maps can be also established (Corollary 4.4). These topics are treated in Sect. 4.

Another class of rotational symmetric distances is that of multiradial distances, that are explored in Sect. 5. Such distances were used in [23] to relate the Hausdorff measure to the spherical measure of horizontal submanifolds. The next theorem provides an area formula with multiradial distance for new classes of submanifolds. The area formula (1.4) also yields a simpler form for the spherical measure of curves with respect to a multiradial distance, improving the results of [17]. 
Theorem 1.3 (Area formulas for multiradial distances) Let $\mathbb{G}$ be a homogeneous group with a multiradial distance $d$ and let $\Sigma \subset \mathbb{G}$ be a $C^{1}$ smooth $\mathrm{n}$-dimensional submanifold of degree N. Suppose that one of the following conditions hold.

(1) The step of $\mathbb{G}$ is two, every point of maximum degree is algebraically regular and points of lower degree are $\mathcal{S}^{\mathrm{N}}$-negligible.

(2) The submanifold $\Sigma$ is one dimensional.

We denote by $\mathcal{F}$ the family of all homogeneous subgroups of topological dimension $\mathrm{n}$ and Hausdorff dimension $\mathrm{N}$. Thus, in both of the previous cases, $d$ is rotationally symmetric with respect to $\mathcal{F}$ and we denote by $\omega_{d}(\mathcal{F})$ the constant spherical factor. Defining $\mathcal{S}_{d}^{\mathrm{N}}=\omega_{d}(\mathcal{F}) \mathcal{S}^{\mathrm{N}}$, for any Borel set $B \subset \Sigma$ the following area formula holds

$$
\mathcal{S}_{d}^{\mathrm{N}}(B)=\int_{B}\left\|\tau_{\Sigma, \mathrm{N}}^{\tilde{g}}(p)\right\|_{g} d \sigma_{\tilde{g}}(p) .
$$

Finally, we study the spherical factor in the important case where the metric unit ball is convex (Sect. 6). This condition is satisfied by important distances, like the CyganKorányi distance, the distance $d_{\infty}$ of [10], and all homogeneous distances arising from the construction of [13, Theorem 2].

Theorem 1.4 If $d$ is a homogeneous distance whose metric unit ball $\mathbb{B}(0,1)$ is convex and $N \subset \mathbb{G}$ is an $\mathrm{n}$-dimensional vertical subgroup of $\mathbb{G}$, then

$$
\beta_{d}(N)=\mathcal{H}_{|\cdot|}^{\mathrm{n}}(N \cap \mathbb{B})
$$

The main tool to prove this theorem is a concavity property of the area of "parallel sections" of convex sets, given in Theorem 6.3. The intersections with the metric unit ball are obtained by translated subspaces of arbitrarily fixed codimension. We believe this result is certainly well known from the Brunn-Minkowski theory of convex bodies. However, due to its importance for our purposes, we also provide its proof.

Formula (1.5) not only simplifies the computation of the spherical factor, but it has also applications in the study of the equality between centered Hausdorff measure and spherical measure. This question in the setting of stratified groups was posed in [9]. The right hand side of (1.5) can be proved to represent the expression of an upper centered density, where $N$ is the homogeneous tangent space. Then we are lead to the equality between Federer density, [21], and upper centered density [7, 2.10.19]. This in turn yields the equality between spherical measure and centered Hausdorff measure. The Q-dimensional spherical measure in $\mathbb{G}$ (where the Hausdorff dimension of $\mathbb{G}$ is Q) always coincides with the Q-dimensional centered Hausdorff measure, according to [9, Corollary 4.13]. However, in general these two measures may differ, [9,21].

As a consequence of (1.5) in the case of one codimensional vertical subgroups, combining [9, (4.22)], [9, (4.23)] and [22, Theorem 1.3], we obtain that the spherical measure equals the centered Hausodorff measure of dimension $Q-1$ on all $\mathbb{G}$-rectifiable sets. This result extends [9, Theorem 4.28] to all homogeneous distances whose metric unit ball is convex. Another application of Theorem 1.4 is for low codimensional $\mathbb{H}$-regular surfaces in Heisenberg groups $\mathbb{H}^{n}$. When the metric unit ball is convex, formula (1.5) was used in [5] to prove that spherical measure and centered 
Hausdorff measure coincide on low codimensional $\mathbb{H}$-regular surfaces. From [23, (1.7)], assuming that the metric unit ball is convex and combining (1.5), the centered blow-up of [25, Theorem 1.1] and [9, Theorem 3.1], we obtain the equality between centered Hausdorff measure and spherical measure also on all transversal submanifolds. Similar applications of (1.3) can be obtained for multiradial distances, starting from Theorems 5.3 and 5.5.

\section{Basic Notions}

A simply connected real and finite dimensional nilpotent Lie group can be regarded as a linear space $\mathbb{G}$ equipped with a Lie product and a polynomial group operation arising from the Baker-Campbell-Hausdorff formula. If we add the assumption that $\mathbb{G}=H^{1} \oplus \cdots \oplus H^{\iota}$ and

$$
\left[H^{i}, H^{j}\right] \subset H^{i+j}
$$

for all $i, j \geq 0$ and $H^{l}=0$ for all $l>\iota$, then we say that $\mathbb{G}$ is a graded group. We have the grading

$$
\operatorname{Lie}(\mathbb{G})=\mathcal{V}_{1} \oplus \cdots \oplus \mathcal{V}_{\iota}, \quad\left[\mathcal{V}_{i}, \mathcal{V}_{j}\right] \subset \mathcal{V}_{i+j}
$$

for all integers $i, j \geq 0$ and $\mathcal{V}_{j}=\{0\}$ for all $j>\iota$, with $\mathcal{V}_{\iota} \neq\{0\}$. The integer $\iota \geq 1$ is called the step of the group. We introduce dilations $\delta_{r}: \mathbb{G} \rightarrow \mathbb{G}$ to be linear mappings such that

$$
\delta_{r}(p)=r^{i} p
$$

for each $p \in H^{i}, r>0$ and $i=1, \ldots, \iota$. According to this homogeneity property of dilations, elements of $H^{i}$ can be called homogeneous vectors, having degree $i$. The graded nilpotent Lie group $\mathbb{G}$ equipped with intrinsic dilations is called homogeneous group, [8].

We may further identify $\mathbb{G}$ with the tangent space $T_{0} \mathbb{G}$ at the origin 0 , getting a canonical isomorphism between $H^{j}$ and $\mathcal{V}_{j}$, that associates to each $v \in H^{j}$ the unique left invariant vector field $X \in \mathcal{V}_{j}$ such that $X(0)=v$. If we think of $\mathbb{G}$ as equipped with a Lie product inducing a Lie algebra structure, we get the following Baker-Campbell-Hausdorff formula

$$
x y=\sum_{j=1}^{\iota} c_{j}(x, y)=x+y+\frac{[x, y]}{2}+\sum_{j=3}^{\iota} c_{j}(x, y)
$$

with $x, y \in \mathbb{G}$, where $c_{j}$ are suitably defined polynomials, [30]. We refer to (2.2) in short as $\mathrm{BCH}$ formula.

In the sequel q denotes the linear dimension of $\mathbb{G}$. Linear subspaces $S$ of $\mathbb{G}$ that satisfy $\delta_{r}(S) \subset S$ for every $r>0$ are called homogeneous subspaces. It is not 
difficult to observe that for a homogeneous subspace $S \subset \mathbb{G}$ we have the characterizing condition

$$
S=S_{1} \oplus \cdots \oplus S_{\iota}
$$

where each $S_{j}$ is a subspace of $H^{j}$. If a homogeneous subspace has in addition the structure of Lie subgroup, then it is called homogeneous subgroup.

A homogeneous distance $d$ on a graded nilpotent Lie group $\mathbb{G}$ is a left invariant distance with $d\left(\delta_{r} x, \delta_{r} y\right)=r d(p, q)$ for all $p, q \in \mathbb{G}$ and $r>0$. Open and closed balls are defined respectively as follows

$$
B(p, r)=\{q \in \mathbb{G}: d(q, p)<r\} \text { and } \mathbb{B}(p, r)=\{q \in \mathbb{G}: d(q, p) \leq r\} .
$$

The homogeneous norm associated to a homogeneous distance $d$ is defined as

$$
\|x\|=d(x, 0)
$$

for all $x \in \mathbb{G}$. When the graded nilpotent Lie group is equipped with the corresponding dilations, along with a homogeneous norm, is called homogeneous group. A graded basis $\left(e_{1}, \ldots, e_{\mathrm{q}}\right)$ of a homogeneous group $\mathbb{G}$ is a basis of vectors such that

$$
\left(e_{\mathrm{m}_{j-1}+1}, e_{\mathrm{m}_{j-1}+2}, \ldots, e_{\mathrm{m}_{j}}\right)
$$

is a basis of $H^{j}$ for each $j=1, \ldots, \iota$, where

$$
\mathrm{m}_{j}=\sum_{i=1}^{j} \mathrm{~h}_{i} \text { and } \mathrm{h}_{j}=\operatorname{dim} H^{j}
$$

we have set $\mathrm{m}_{0}=0$. We also set $\mathrm{m}=\mathrm{m}_{1}$ and observe that $\mathrm{m}_{\iota}=\mathrm{q}$. A graded basis provides the associated graded coordinates $x=\left(x_{1}, \ldots, x_{\mathrm{q}}\right) \in \mathbb{R}^{\mathrm{q}}$, then defining the unique element $p=\sum_{j=1}^{\mathrm{q}} x_{j} e_{j} \in \mathbb{G}$. It is easy to realize that one can always equip a homogeneous subgroup with graded coordinates. We fix throughout the paper a graded left invariant Riemannian metric $g$ on the homogeneous group $\mathbb{G}$, that automatically induces a scalar product on $T_{0} \mathbb{G}$. By the identification of $\mathbb{G}$ with $T_{0} \mathbb{G}$ we also have a fixed scalar product on $\mathbb{G}$.

The fact that our left invariant Riemannian metric $g$ is "graded" means that the induced scalar product on $\mathbb{G}$ is graded, that is, all subspaces $H^{i}$ with $i=1, \ldots, \iota$ are orthogonal to each other. With a slight abuse of notation, both the norm on $\mathbb{G}$ and the norm arising from the Riemannian metric $g$ on tangent spaces are denoted by the same symbol $|\cdot|$. We refer to the norm on $\mathbb{G}$ as arising from a graded scalar product, hence all subspaces $H^{i}$ are orthogonal to each other. Clearly when a graded scalar product is fixed, we can find a graded basis that is also orthonormal with respect to this scalar product.

We close this section introducing the Carathéodory measure, which includes both spherical measure and Hausdorff measure, that are used throughout the paper. 
Let $\mathcal{F} \subset \mathcal{P}(\mathbb{G})$ denote a nonempty family of closed subsets and fix $\alpha>0$. For any $\delta>0$ and $E \subset \mathbb{G}$, we define

$$
\phi_{\delta}^{\alpha}(E)=\inf \left\{\sum_{j=0}^{\infty} \frac{\operatorname{diam}\left(B_{j}\right)^{\alpha}}{2^{\alpha}}: E \subset \bigcup_{j \in \mathbb{N}} B_{j}, \operatorname{diam}\left(B_{j}\right) \leq \delta, B_{j} \in \mathcal{F}\right\},
$$

where the diameter $\operatorname{diam}\left(B_{j}\right)$ is computed with respect to the distance $d$ on $\mathbb{G}$. When $\mathcal{F}$ is the family of closed balls $\mathcal{F}_{b}$, then we set

$$
\mathcal{S}^{\alpha}(E)=\sup _{\delta>0} \phi_{\delta}^{\alpha}(E)
$$

to be the $\alpha$-dimensional spherical measure of $E$. In the case $\mathcal{F}$ is the family of all closed sets and $k \in\{1,2, \ldots, \mathrm{q}-1\}$, for any $A \subset \mathbb{G}$ we define the Hausdorff measure

$$
\mathcal{H}_{|\cdot|}^{k}(A)=\mathcal{L}^{k}\left(\left\{x \in \mathbb{R}^{k}: d_{E}(x, 0) \leq 1\right\}\right) \sup _{\delta>0} \phi_{\delta,|\cdot|}^{k}(A)
$$

where $\mathcal{L}^{k}$ denotes the Lebesgue measure on $\mathbb{R}^{k}$ and $d_{E}$ denotes the Euclidean distance on $\mathbb{R}^{k}$. The outer measure $\phi_{\delta,|\cdot|}^{k}$ refers to the norm $|\cdot|$ defined by the fixed graded scalar product on $\mathbb{G}$.

\section{Vertically Symmetric Distances}

The main object of this section is the proof of Theorem 1.1. We start by recalling the notion of vertical subgroup and of transversal submanifold. More information can be found in [25] and [23, Sect. 5].

Definition 3.1 A homogeneous subgroup $N \subset \mathbb{G}$ is vertical if we have a positive integer $\ell \leq \iota$ and a linear subspace $N_{\ell} \subset H^{\ell}$ such that

$$
N=N_{\ell} \oplus H^{\ell+1} \oplus \cdots \oplus H^{\iota}
$$

We denote by $\mathcal{N}_{\mathrm{n}}$ the family of all $\mathrm{n}$-dimensional vertical subgroups.

A transversal submanifold in $\mathbb{G}$ is a $C^{1}$ smooth submanifold having at least one point where the homogeneous tangent space is a vertical subgroup.

Denoting by $\mathrm{n}$ the dimension of the vertical subgroup $N$, we can find an explicit formula for the integer $\ell$ appearing in (3.1). We denote this integer by $\ell_{\mathrm{n}}$ since it depends on the dimension of $N$. One can easily check that

$$
\left\{\begin{array}{ll}
\ell_{\mathrm{n}}=\iota & \text { if } 1 \leq \mathrm{n} \leq \operatorname{dim} H^{\iota} \\
\sum_{j=\ell_{\mathrm{n}}+1}^{\iota} \operatorname{dim} H^{j}<\mathrm{n} \leq \sum_{j=\ell_{\mathrm{n}}}^{\iota} \operatorname{dim} H^{j} & \text { if } \operatorname{dim} H^{\iota}<\mathrm{n} \leq \mathrm{q}
\end{array} .\right.
$$


An equivalent definition for $\ell_{\mathrm{n}}$ is to see it as the minimal degree of homogeneous vectors belonging to $N$. Associated to $\ell_{n}$, we also introduce the integer

$$
\mathrm{r}_{\mathrm{n}}:=\left\{\begin{array}{lr}
\mathrm{n} & \text { if } 1 \leq \mathrm{n} \leq \operatorname{dim} H^{\iota} \\
\mathrm{n}-\sum_{j=\ell_{\mathrm{n}}+1}^{\iota} \operatorname{dim} H^{j} & \text { if } \operatorname{dim} H^{\iota}<\mathrm{n} \leq \mathrm{q}
\end{array},\right.
$$

where $1 \leq \mathrm{n} \leq \mathrm{q}$ and clearly by definition $\mathrm{r}_{\mathrm{n}} \geq 1$. We finally observe that the integer

$$
\mathrm{Q}_{\mathrm{n}}=\ell_{\mathrm{n}} \mathrm{r}_{\mathrm{n}}+\sum_{j=\ell_{\mathrm{n}}+1}^{\iota} j \operatorname{dim} H^{j}
$$

precisely corresponds to the Hausdorff dimension of $N$ with respect to $d$. It can be proved that (3.4) is the Hausdorff dimension of any transversal submanifold of dimension $n$, see [25].

To introduce the notion of n-vertically symmetric distance, we define the following class of isometries

$$
\mathcal{O}_{\mathrm{n}}(\mathcal{F})=\left\{T: \mathbb{G} \rightarrow \mathbb{G} \mid T \text { is linear, }\left.T\right|_{H^{j}}=\operatorname{Id}_{H^{j}} \text { for all } j \neq \ell_{\mathrm{n}},\left.T\right|_{H^{\ell_{\mathrm{n}}}} \in \mathcal{F}\right\}
$$

where we have taken into account that the subspaces $H^{i}$ and $H^{j}$ of $\mathbb{G}$ are orthogonal for $i \neq j$ and $\mathcal{F}$ is a family of isometries of $H^{\ell_{\mathrm{n}}}$. We also introduce the subspaces

$$
U_{\mathrm{n}}=H^{1} \oplus \cdots \oplus H^{\ell_{\mathrm{n}}-1} \quad \text { and } \quad V_{\mathrm{n}}=H^{1} \oplus \cdots \oplus H^{\ell_{\mathrm{n}}}
$$

where $U_{\mathrm{n}}=\{0\}$ for $\ell_{\mathrm{n}}=1$.

Definition 3.2 (n-vertically symmetric distance) Let $1 \leq \mathrm{n} \leq \mathrm{q}$ and consider $\ell_{\mathrm{n}}$ as in (3.2) and $r_{n}$ as in (3.3). We introduce the integer

$$
J=\left\{\begin{array}{l}
0 \text { if } r_{n}=\operatorname{dim} H^{\ell_{n}} \\
r_{n} \text { if } r_{n}<\operatorname{dim} H^{\ell_{n}}
\end{array} .\right.
$$

If $J=0$, then we say that $d$ is n-vertically symmetric. In the case $j>0$, we say that $d$ is n-vertically symmetric if the following conditions hold.

- There exists a family $\mathcal{F}$ of isometries of $H^{\ell_{\mathrm{n}}}$ such that for every couple of $\mathrm{J}$ dimensional subspaces $S_{1}, S_{2} \subset H^{\ell_{\mathrm{n}}}$, there exists $J \in \mathcal{F}$ that satisfies

$$
J\left(S_{1}\right)=S_{2}
$$

- Let $U_{\mathrm{n}}$ and $V_{\mathrm{n}}$ be as in (3.6) and let $P_{V_{\mathrm{n}}}: \mathbb{G} \rightarrow V_{\mathrm{n}}$ and $P_{U_{\mathrm{n}}}: \mathbb{G} \rightarrow U_{\mathrm{n}}$ be the orthogonal projections onto $V_{\mathrm{n}}$ and $U_{\mathrm{n}}$, respectively. We consider $\mathcal{O}_{\mathrm{n}}(\mathcal{F})$ defined in (3.5) by our family of isometries $\mathcal{F}$. Then we have: 
(1) $P_{V_{\mathrm{n}}}(\mathbb{B}(0,1))=\mathbb{B}(0,1) \cap V_{\mathrm{n}}=\left\{v \in V_{\mathrm{n}}: \psi\left(P_{U_{\mathrm{n}}}(v),\left|P_{H^{\ell_{\mathrm{n}}}}(v)\right|\right) \leq r_{0}\right\}$ for some $r_{0}>0$ and $\psi: U_{\mathrm{n}} \times[0,+\infty) \rightarrow[0,+\infty)$ is such that $\psi(u, \cdot)$ is monotone nondecreasing for all $u \in U_{\mathrm{n}}$,

(2) $T(\mathbb{B}(0,1))=\mathbb{B}(0,1)$ for all $T \in \mathcal{O}_{\mathrm{n}}(\mathcal{F})$.

The metric unit ball $\mathbb{B}(0,1)$ is with respect to $d$ and we have assumed that when $\ell_{\mathrm{n}}=1$, then $\psi$ is any monotone nondecreasing function from $[0,+\infty)$ to $[0,+\infty)$.

Remark 3.1 The previous definition when $\mathrm{n}=\mathrm{q}-1, \ell_{\mathrm{n}}=1$ and $\psi(t)=t$, yields the notion of $H^{1}$-vertical symmetry introduced in [22, Definition 6.1]. In this definition the transitivity of isometries of $H^{1}$ corresponds to (3.7) for $J=\operatorname{dim} H^{1}-1$. In a few words, any $H^{1}$-vertically symmetric distance is also a $(\mathrm{q}-1)$-vertically symmetric distance.

Example 3.2 In any H-type group with direct decomposition $\mathbb{G}=H^{1} \oplus H^{2}$, the well known Cygan-Korányi norm

$$
\|x\|=\sqrt[4]{\left|x_{1}\right|^{4}+16\left|x_{2}\right|^{2}}
$$

where $\left(x_{1}, x_{2}\right) \in H^{1} \times H^{2}$ and $x=x_{1}+x_{2}$, yields the associated homogeneous distance $d(x, y)=\left\|x^{-1} y\right\|$, see [6]. It can be checked that $d$ is $p$-vertically symmetric for any $p=1, \ldots, \operatorname{dim} \mathbb{G}-1$. This will be also a consequence of Proposition 5.1.

The next lemma provides a factorization of a homogeneous group $\mathbb{G}$ with respect to a vertical subgroup $N$, although a complementary subgroup $V$ such that $V \oplus N=\mathbb{G}$ may not exist. We simply consider $V$ as a homogeneous subspace of $\mathbb{G}$.

Lemma 3.3 If $\mathbb{G}$ is a homogeneous group, $V \subset \mathbb{G}$ is a homogeneous subspace and $N \subset \mathbb{G}$ is a vertical subgroup such that $V \oplus N=\mathbb{G}$, then the mapping

$$
V \times N \rightarrow \mathbb{G}, \quad(v, h) \rightarrow v h
$$

is an analytic diffeomorphism. Furthermore, its inverse mapping $T: \mathbb{G} \rightarrow V \times N$ is defined by

$$
T(x)=\left(P_{V}(x), \Pi_{N}(x)\right)
$$

where $P_{V}: \mathbb{G} \rightarrow V$ is the linear projection onto $V$ with respect to the direct sum $\mathbb{G}=V \oplus N$ and $\Pi_{N}(x)=P_{V}(x)^{-1} x$.

Proof The mapping $F(v, h)=v h$ has the property that

$$
\left.\partial_{v} F(0,0)\right|_{V}=\operatorname{Id}_{V} \text { and }\left.\partial_{h} F(0,0)\right|_{N}=\operatorname{Id}_{N}
$$

so the assumption that $V \oplus N=\mathbb{G}$ implies that $d F(0,0)$ is invertible and $F$ is locally invertible around the origin. The homogeneity of $F$, i.e.

$$
F\left(\delta_{r} v, \delta_{r} h\right)=\delta_{r} F(h, v)
$$


shows that $F$ is surjective. We now consider

$$
v h=w k,
$$

where $v, w \in V$ and $h, k \in N$. By the $\mathrm{BCH}$ formula (2.2), we have

$$
v+h+\sum_{j=2}^{\iota} c_{j}(v, h)=w+k+\sum_{j=2}^{\iota} c_{j}(w, k)
$$

where the fact that $N$ is vertical gives

$$
h+\sum_{j=2}^{\iota} c_{j}(v, h) \in N \quad \text { and } \quad k+\sum_{j=2}^{\iota} c_{j}(w, k) \in N
$$

Applying $P_{V}$ to the equality (3.8), it follows that

$$
v=P_{V}(v h)=P_{V}(w k)=w .
$$

We have shown that any element $x \in \mathbb{G}$ can be uniquely written as the product

$$
P_{V}(x) \Pi_{N}(x),
$$

therefore concluding the proof.

Lemma 3.4 If $\mathbb{G}$ is a homogeneous group, $V \subset \mathbb{G}$ is a homogeneous subspace and $N \subset \mathbb{G}$ is a vertical subgroup such that $V \oplus N=\mathbb{G}$, then for every $v \in V$ we have

$$
v+N=v N
$$

Proof From (2.2) and the fact that $N$ is an ideal with respect to the Lie algebra structure of $\mathbb{G}$, there holds

$$
v n=v+n+\sum_{j=2}^{\iota} c_{j}(v, n) \in v+N
$$

being $c_{j}(v, n) \in N$ for every $j=2, \ldots, \iota$. This shows that $v N \subset v+N$. Conversely, considering $v+n \in \mathbb{G}$ and applying Lemma 3.3 we get

$$
v+n=P_{V}(v+n) \Pi_{N}(v+n)=v \Pi_{N}(v+n)=v \tilde{n}
$$

where $\tilde{n}=\Pi_{N}(v+n) \in N$. We have then established the opposite inclusion.

An important feature of vertical subgroups is the following left invariance property of the Hausdorff measure, once we consider our fixed graded scalar product on $\mathbb{G}$. 
Lemma 3.5 If $\mathbb{G}$ is a homogeneous group and $N \subset \mathbb{G}$ is an $\mathrm{n}$-dimensional vertical subgroup, then for every $p \in \mathbb{G}$ and every measurable set $A \subset N$, we have

$$
\mathcal{H}_{|\cdot|}^{\mathrm{n}}(A)=\mathcal{H}_{|\cdot|}^{\mathrm{n}}\left(l_{p}(A)\right)
$$

where $l_{p}: \mathbb{G} \rightarrow \mathbb{G}$ denotes the left translation by $p$.

Proof We consider a graded basis $\left(e_{1}, \ldots, e_{\mathrm{q}}\right)$, such that

$$
V=\operatorname{span}\left\{e_{1}, \ldots, e_{\mathrm{q}-\mathrm{n}}\right\} \text { and } N=\operatorname{span}\left\{e_{\mathrm{q}-\mathrm{n}+1}, \ldots, e_{\mathrm{q}}\right\}
$$

and the associated graded coordinates in $\mathbb{G}$, setting

$$
p=\sum_{j=1}^{\mathrm{q}} x_{j} e_{j} \text { and } n=\sum_{j=1}^{\mathrm{n}} \zeta_{j} e_{\mathrm{q}-\mathrm{n}+j} \in N .
$$

We express the left translation explicitly

$$
l_{p} n=\sum_{j=1}^{\mathrm{q}-\mathrm{n}} x_{j} e_{j}+\sum_{j=\mathrm{q}-\mathrm{n}+1}^{\mathrm{q}}\left(x_{j}+\zeta_{j-\mathrm{q}+\mathrm{n}}\right) e_{j}+\sum_{j=\mathrm{m}+1}^{\mathrm{q}} c_{j}\left(\bar{x}^{d_{j}-1}, \bar{\zeta}^{d_{j}-1}\right) e_{j} .
$$

According to the Baker-Campbell-Hausdorff formula, the functions $c_{j}$ are polynomials that only depends on variables of degree less than $d_{j}$. We have defined

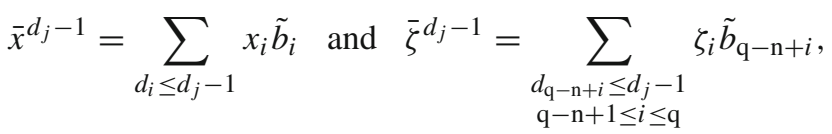

where $\left(\tilde{b}_{1}, \ldots, \tilde{b}_{\mathrm{q}}\right)$ is the canonical basis of $\mathbb{R}^{\mathrm{q}}$. Let us remark that $\bar{\zeta}^{d_{j}-1}=0$ for $d_{j} \leq d_{\mathrm{q}-\mathrm{n}+1}$. We decompose $p$ into the sum

$$
p=v+w, \quad \text { where } v=\sum_{j=1}^{\mathrm{q}-\mathrm{n}} x_{j} e_{j} \text { and } w=\sum_{j=\mathrm{q}-\mathrm{n}+1}^{\mathrm{q}} x_{j} e_{j}
$$

With this notation formula (3.9) gives $p N=v+N$. Since $v \notin N$, this decomposition improves (3.11), giving

$$
l_{p} n=\sum_{j=1}^{\mathrm{q}-\mathrm{n}} x_{j} e_{j}+\sum_{j=\mathrm{q}-\mathrm{n}+1}^{\mathrm{q}}\left(x_{j}+\zeta_{j}\right) e_{j}+\sum_{j=k_{\mathrm{n}}}^{\mathrm{q}} c_{j}\left(\bar{x}^{d_{j}-1}, \bar{\zeta}^{d_{j}-1}\right) e_{j},
$$


with $k_{\mathrm{n}}=\max \{\mathrm{q}-\mathrm{n}+1, \mathrm{~m}+1\}$. We now consider the projection

$$
T: v+N \rightarrow \mathbb{R}^{\mathrm{n}}, \quad v+\sum_{j=\mathrm{q}-\mathrm{n}+1}^{\mathrm{q}} \zeta_{j} e_{j} \longrightarrow \sum_{j=\mathrm{q}-\mathrm{n}+1}^{\mathrm{q}} \zeta_{j} b_{j-\mathrm{q}+\mathrm{n}}
$$

where $\left(b_{1}, \ldots, b_{\mathrm{n}}\right)$ is the canonical basis of $\mathbb{R}^{\mathrm{n}}$ and $J: \mathbb{R}^{\mathrm{n}} \rightarrow N$, defined as

$$
J\left(\zeta_{1}, \ldots, \zeta_{\mathrm{n}}\right)=\sum_{j=1}^{\mathrm{n}} \zeta_{j} e_{\mathrm{q}-\mathrm{n}+j}
$$

The composition $F: \mathbb{R}^{\mathrm{n}} \rightarrow \mathbb{R}^{\mathrm{n}}$ defined as $F=T \circ l_{p} \circ J$ can be written as follows

$$
F(\zeta)=\sum_{j=1}^{\mathrm{n}}\left(x_{\mathrm{q}-\mathrm{n}+j}+\zeta_{j}\right) b_{j}+\sum_{j=k_{\mathrm{n}}-\mathrm{q}+\mathrm{n}}^{\mathrm{n}} c_{\mathrm{q}-\mathrm{n}+j}\left(\bar{x}^{d_{\mathrm{q}-\mathrm{n}+j}-1}, \bar{\zeta}^{d_{\mathrm{q}-\mathrm{n}+j}-1}\right) b_{j} .
$$

As a consequence of the previous formulas, for every $j, l=1, \ldots, \mathrm{n}$ we get

$$
\frac{\partial F_{j}}{\partial \zeta_{l}}=\delta_{l}^{j}+\frac{\partial c_{\mathrm{q}-\mathrm{n}+j}\left(\bar{x}^{\mathrm{q}-\mathrm{n}+j}, \cdot\right)}{\partial \zeta_{l}}
$$

In the special case $l \geq j$, due to (3.12) the function $\zeta \rightarrow c_{\mathrm{q}-\mathrm{n}+j}\left(\bar{x}^{\mathrm{q}-\mathrm{n}+j}, \zeta\right)$ only depends on $\zeta_{i}$ with $d_{\mathrm{q}-\mathrm{n}+i} \leq d_{\mathrm{q}-\mathrm{n}+j}-1$, therefore $i<j \leq l$. We have proved that

$$
\frac{\partial F_{j}}{\partial \zeta_{l}}=\delta_{i}^{j} \quad \text { whenever } l \geq j
$$

hence the Jacobian of $F$ is one. Since both $T$ and $J$ are isometries one easily observes that image measures satisfy

$$
T_{\sharp} \mathcal{H}_{|\cdot|}^{\mathrm{n}}=\mathcal{L}^{\mathrm{n}} \quad \text { and } \quad J_{\sharp} \mathcal{L}^{\mathrm{n}}=\mathcal{H}_{|\cdot|}^{\mathrm{n}} \cdot
$$

Since $F$ preserves the Lebesgue measure $\mathcal{L}^{\mathrm{n}}$, the following equalities conclude the proof, that is

$$
\begin{aligned}
\mathcal{H}_{|\cdot|}^{\mathrm{n}}\left(l_{p}(A)\right) & =T_{\sharp} \mathcal{H}_{|\cdot|}^{\mathrm{n}}\left(F \circ J^{-1}(A)\right)=\mathcal{L}^{n}\left(F\left(J^{-1}(A)\right)\right) \\
& =\mathcal{L}^{n}\left(J^{-1}(A)\right)=J_{\sharp} \mathcal{L}^{\mathrm{n}}(A)=\mathcal{H}_{|\cdot|}^{\mathrm{n}}(A) .
\end{aligned}
$$

The previous results allow us to establish our first main result. 


\subsection{Proof of Theorem 1.1}

First of all, we consider the integers $\ell_{\mathrm{n}}$ and $\mathrm{r}_{\mathrm{n}}$ defined in (3.2) and (3.3), respectively. If $\mathrm{r}_{\mathrm{n}}=\operatorname{dim} H^{\ell_{\mathrm{n}}}$, then $J=0$ in Definition 3.2 and $d$ is automaticaly n-vertically symmetric. Thus, the only n-dimensional vertical subgroup is

$$
N_{0}=H^{\ell_{\mathrm{n}}} \oplus \cdots \oplus H^{\iota}
$$

and the spherical factor is obviously constant and equal to $\beta\left(d, N_{0}\right)$. Let us consider the case $0<j=\mathrm{r}_{\mathrm{n}}<\operatorname{dim} H^{\ell_{\mathrm{n}}}$, where

$$
\mathrm{n}=J+\mathrm{h}_{\ell_{\mathrm{n}}+1}+\cdots+h_{\iota} .
$$

To simplify notation, in the rest of the proof we will write $\ell$ in place of $\ell_{\mathrm{n}}$. We fix $z \in \mathbb{B}(0,1)$ and consider two arbitrary n-dimensional vertical subgroups

$$
N_{1}=S_{1} \oplus W \quad \text { and } \quad N_{2}=S_{2} \oplus W
$$

where $S_{1}$ and $S_{2}$ are J-dimensional subspaces of $H^{\ell}$ and

$$
W=H^{\ell+1} \oplus \cdots \oplus H^{\iota} .
$$

By the n-vertical symmetry of $d$, there exists an isometry $J: H^{\ell} \rightarrow H^{\ell}$ with

$$
J\left(S_{1}\right)=S_{2}
$$

This defines the isometry $T: \mathbb{G} \rightarrow \mathbb{G}$ such that

$$
\left.T\right|_{H^{j}}=\operatorname{Id}_{H^{j}} \text { and }\left.T\right|_{H^{\ell}}=J
$$

for each $j \neq \ell$. We now define the subspace

$$
V_{1}=H^{1} \oplus \cdots \oplus H^{\ell-1} \oplus Z_{1}
$$

such that $Z_{1}$ is orthogonal to $S_{1}$ and

$$
Z_{1} \oplus S_{1}=H^{\ell}
$$

We consider the orthogonal projection $P_{V_{1}}: \mathbb{G} \rightarrow V_{1}$, that is also the linear projection associated to the direct sum

$$
\mathbb{G}=V_{1} \oplus N_{1} .
$$

Defining the nonlinear projection

$$
\Pi_{N_{1}}(x)=P_{V_{1}}(x)^{-1} x
$$


Lemma 3.3 ensures the following unique product decomposition

$$
z^{-1}=P_{V_{1}}\left(z^{-1}\right) \Pi_{N_{1}}\left(z^{-1}\right) \text { with } \Pi_{N_{1}}\left(z^{-1}\right) \in N_{1} \text {. }
$$

Setting $P_{V_{1}}\left(z^{-1}\right)=v_{1}, \Pi_{N_{1}}\left(z^{-1}\right)=h_{1}$ and taking into account (3.10), (3.13) and (3.9), we get

$$
\mathcal{H}_{|\cdot|}^{\mathrm{n}}\left(\mathbb{B}(z, 1) \cap N_{1}\right)=\mathcal{H}_{|\cdot|}^{\mathrm{n}}\left(\mathbb{B}(0,1) \cap\left(v_{1}+N_{1}\right)\right)
$$

Since the previously defined mapping $T$ belongs to $\mathcal{O}$, in view of the property (2) of Definition 3.2, we obtain that

$$
\mathcal{H}_{|\cdot|}^{\mathrm{n}}\left(\mathbb{B}(z, 1) \cap N_{1}\right)=\mathcal{H}_{|\cdot|}^{\mathrm{n}}\left(\mathbb{B}(0,1) \cap\left(T v_{1}+T\left(N_{1}\right)\right)\right)
$$

It is not difficult to realize that

$$
T\left(N_{1}\right)=T\left(S_{1} \oplus W\right)=J\left(S_{1}\right) \oplus W=S_{2} \oplus W=N_{2},
$$

due to the definition of $T$ and the inclusions $S_{1} \subset H^{\ell}$. We have proved that

$$
\mathcal{H}_{|\cdot|}^{\mathrm{n}}\left(\mathbb{B}(z, 1) \cap N_{1}\right)=\mathcal{H}_{|\cdot|}^{\mathrm{n}}\left(\mathbb{B}(0,1) \cap\left(T v_{1}+N_{2}\right)\right)
$$

We wish to check whether $\left(T v_{1}\right)^{-1}$ is a suitable element of $\mathbb{B}(0,1)$. To do this, we define the subspace

$$
V=H^{1} \oplus \cdots \oplus H^{\ell}
$$

and consider the orthogonal decompositions

$$
z^{-1}=v_{1}+\eta_{1}=v+\eta
$$

where $P_{V}\left(z^{-1}\right)=v \in V, v_{1}$ and $v$ are orthogonal to $\eta_{1} \in N_{1}$ and $\eta \in W$, respectively. The previous equality gives

$$
s_{1}=v-v_{1}=\eta_{1}-\eta \in V \cap\left(S_{1} \oplus W\right)=S_{1} \subset H^{\ell},
$$

hence $v_{1}$ and $s_{1}$ are orthogonal. We write the orthogonal decomposition

$$
v_{1}=w_{1}+z_{1} \quad \text { with } w_{1} \in H^{1} \oplus \cdots \oplus H^{\ell-1} \text { and } z_{1} \in Z_{1},
$$

therefore $v=w_{1}+z_{1}+s_{1}$. Since $s_{1}$ is orthogonal to $w_{1}$ and to $z_{1}$, we get

$$
\left|P_{H^{\ell}} v\right|=\left|z_{1}+s_{1}\right|=\sqrt{\left|z_{1}\right|^{2}+\left|s_{1}\right|^{2}} \geq\left|z_{1}\right|
$$


By the property (1) of Definition 3.2, since

$$
P_{V}\left(z^{-1}\right)=v \in P_{V}(\mathbb{B}(0,1))=\mathbb{B}(0,1) \cap V,
$$

we have $\psi(u, t) \geq 0$ that is monotone nondecreasing with respect to $t$ and such that

$$
v \in \mathbb{B}(0,1) \cap V=\left\{y \in V: \psi\left(P_{U}(y),\left|P_{H^{\ell}}(y)\right|\right) \leq r_{0}\right\},
$$

where $U=H^{1} \oplus \cdots \oplus H^{\ell-1}$. From the monotonicity of $\psi$ we have

$$
\psi\left(P_{U}\left(v_{1}\right),\left|P_{H^{\ell}}\left(v_{1}\right)\right|\right)=\psi\left(P_{U}(v),\left|z_{1}\right|\right) \leq \psi\left(P_{U}(v),\left|P_{H^{\ell}}(v)\right|\right) \leq r_{0} .
$$

We have proved that $v_{1} \in V \cap \mathbb{B}(0,1)$. Moreover, taking into account that

$$
T v_{1}=w_{1}+J z_{1}=P_{U}\left(v_{1}\right)+J z_{1}=P_{U}\left(T v_{1}\right)+J z_{1}
$$

there holds

$\psi\left(P_{U}\left(T v_{1}\right),\left|P_{H^{\ell}}\left(T v_{1}\right)\right|\right)=\psi\left(w_{1},\left|J z_{1}\right|\right)=\psi\left(w_{1},\left|z_{1}\right|\right)=\psi\left(P_{U}\left(v_{1}\right),\left|P_{H^{\ell}}\left(v_{1}\right)\right|\right) \leq r_{0}$.

It follows that

$$
T v_{1} \in \mathbb{B}(0,1) \cap V \subset \mathbb{B}(0,1)
$$

and clearly $v_{0}=\left(T v_{1}\right)^{-1} \in V \cap \mathbb{B}(0,1)$. Due to (3.10), it follows that

$$
\mathcal{H}_{|\cdot|}^{\mathrm{n}}\left(\mathbb{B}\left(v_{0}, 1\right) \cap N_{2}\right)=\mathcal{H}_{|\cdot|}^{\mathrm{n}}\left(\mathbb{B}(0,1) \cap v_{0}^{-1} N_{2}\right)=\mathcal{H}_{|\cdot|}^{\mathrm{n}}\left(\mathbb{B}(0,1) \cap\left(T v_{1}\right) N_{2}\right) .
$$

Since $T$ is an isometry, the images $T v_{1}$ and $N_{2}=T\left(N_{1}\right)$ are orthogonal, being so $v_{1}$ and $N_{1}$. We may apply (3.9), getting

$$
\mathcal{H}_{|\cdot|}^{\mathrm{n}}\left(\mathbb{B}\left(v_{0}, 1\right) \cap N_{2}\right)=\mathcal{H}_{|\cdot|}^{\mathrm{n}}\left(\mathbb{B}(0,1) \cap\left(T v_{1}+N_{2}\right)\right) .
$$

The previous equality joined with (3.14) yields

$$
\mathcal{H}_{|\cdot|}^{\mathrm{n}}\left(\mathbb{B}(z, 1) \cap N_{1}\right)=\mathcal{H}_{|\cdot|}^{\mathrm{n}}\left(\mathbb{B}\left(v_{0}, 1\right) \cap N_{2}\right) \leq \beta_{d}\left(N_{2}\right)
$$

and the arbitrary choice of $z \in \mathbb{B}(0,1)$ yields $\beta_{d}\left(N_{1}\right) \leq \beta_{d}\left(N_{2}\right)$. Exchanging the role of $N_{1}$ with that of $N_{2}$, we are able to conclude the proof.

Remark 3.6 The intriguing aspect of Theorem 1.1 is that the metric unit ball is not assumed to satisfy a strong geometric condition like convexity. The role of convexity is specifically studied in Sect. 6. 
As an example, the sub-Riemannian ball in the Heisenberg group is not convex, but it is 2-vertically symmetric, as already pointed out in [22]. This is also an interesting case where a distance is vertical symmetric, but it does not satisfy the stronger condition of being multiradial. Multiradial distances will be studied in Sect. 5 .

\section{Area and Coarea Formulas in Low Codimension}

The area formula (1.3) for n-vertically symmetric distances implies a simple form of area and coarea formulas in low codimension. Indeed, when $k \leq \mathrm{m}=\operatorname{dim} H^{1}$, the level set of a smooth $\mathbb{R}^{k}$-valued mapping $f$ is a $k$-codimensional transversal submanifold if and only if all the horizontal gradients $\nabla_{H} f_{i}$ of the components $f_{i}$ are independent. Thus, the area formula (1.3) applies, giving the simpler area formulas (4.2), (4.3) and (4.4), and finally leading to the coarea formula of Corollary 4.4.

In this section we always assume $k \leq \mathrm{m}$. Then a $C^{1}$ smooth submanifold of codimension $k$ is non-horizontal if and only if its degree is $\mathrm{Q}-k$. We fix a Riemannian metric $\tilde{g}$ such that its associated volume measure $v \mathrm{l}_{\tilde{g}}$ is left invariant. Considering also our fixed left invariant Riemannian metric $g$, we introduce the associated the linear isomorphisms $\tilde{g}^{*}, g^{*}: T \mathbb{G} \rightarrow T^{*} \mathbb{G}$. In a canonical way, taking wedge products, such mappings generate $\tilde{g}_{k}^{*}, g_{k}^{*}: \Lambda_{k}(T \mathbb{G}) \rightarrow \Lambda^{k}(T \mathbb{G})$. Following the notation of [19], we introduce the unit $k$-normals $\mathbf{n}$ and $\tilde{\mathbf{n}}$ of a non-horizontal submanifold $\Sigma$, that defined with respect to the Riemannian metrics $g$ and $\tilde{g}$, respectively. The horizontal $k$-normal at $p$ with respect to $\tilde{g}$ and $g$ is defined as

$$
\tilde{\mathbf{n}}_{g}(p)=\left(g_{k}^{*}\right)^{-1} \tilde{g}_{k}^{*}(\tilde{\mathbf{n}}(p))
$$

for every $p \in \Sigma$ of degree $\mathrm{Q}-k$. Using the horizontal projection $\pi_{g, H}$ with respect to $g$ ( [19, Definition 3.1]), we set

$$
\tilde{\mathbf{n}}_{g, H}(p)=\pi_{g, H}\left(\tilde{\mathbf{n}}_{g}(p)\right) .
$$

We define the unique geometric constant $c(g, \tilde{g})>0$ such that

$$
c(g, \tilde{g}) \operatorname{vol}_{\tilde{g}}=\operatorname{vol}_{g} .
$$

Since the class of $(\mathrm{q}-k)$-vertically symmetric distances is larger than the one in [19, Sect. 6], using Theorem 1.2 we obtain an area formula with constant spherical factor for a larger family of distances.

Corollary 4.1 Let $1 \leq k \leq \mathrm{m}$ and let $\Sigma \subset \mathbb{G}$ be a $C^{1}$ smooth submanifold of codimension $k$ and degree $\mathrm{Q}-k$. Let $d$ be $(\mathrm{q}-k)$-vertically symmetric, let $\mathcal{N}_{\mathrm{q}-k}$ be the family of all vertical subgroups of topological dimension $\mathrm{q}-k$ and let $\omega_{d}\left(\mathcal{N}_{\mathrm{q}-k}\right)$ be the constant spherical factor associated to this family. We fix any Riemannian metric $\tilde{g}$ whose volume measure is left invariant. We rescale the spherical measure as follows

$$
\mathcal{S}_{d}^{\mathrm{Q}-k}=\omega_{d}\left(\mathcal{N}_{\mathrm{q}-k}\right) \mathcal{S}^{\mathrm{Q}-k}
$$


For every Borel set $B \subset \Sigma$, it follows that

$$
\mathcal{S}_{d}^{\mathrm{Q}-k}(B)=c(g, \tilde{g}) \int_{B}\left\|\tilde{\mathbf{n}}_{g, H}(p)\right\|_{g} d \sigma_{\tilde{g}}(p) .
$$

Proof Being $1 \leq k \leq \mathrm{m}$, we have $\mathrm{Q}_{\mathrm{q}-k}=\mathrm{Q}-k$, where $\mathrm{Q}_{n}$ is given by formula (3.4). Our claim is then a consequence of (1.3) joined with formula (12) of [19].

The spherical measure $\mathcal{S}^{\mathrm{Q}-k}$ appearing in (4.1) is introduced at the end of Sect. 2.

Remark 4.2 It is well known that any $C^{1}$ smooth hypersurface $\Sigma \subset \mathbb{G}$ is automatically a non-horizontal submanifold, therefore (4.2) holds for any $C^{1}$ smooth hypersurface of a homogeneous group $\mathbb{G}$. The local isoperimetric inequality applied to a suitably "small" open subset $U \subset \Sigma$ of $\Sigma$ shows that it must have positive $\mathcal{S}^{\mathrm{Q}-1}$ measure. Since characteristic points are $\mathcal{S}^{\mathrm{Q}-1}$ negligible, [18], the subset $U$ must contain noncharacteristic points. This shows that $\Sigma$ has degree Q -1 .

In any homogeneous group $\mathbb{G}$ equipped with graded coordinates $x_{j}$, the corresponding basis of left invariant vector fields $X_{1}, \ldots, X_{\mathrm{q}}$ has the form

$$
X_{j}=\partial_{x_{j}}+\sum_{d_{l}>d_{j}} a_{j l} X_{l},
$$

and it is automatically assumed to be orthonormal with respect to the fixed left invariant metric $g$. The dual basis of left invariant differential forms has the form

$$
\xi_{j}=d x_{j}+\sum_{d_{l}<d_{j}} b_{j l} d x_{l}
$$

The special form of the left invariant differential forms $\xi_{j}$ implies that

$$
\xi_{1} \wedge \xi_{2} \wedge \cdots \wedge \xi_{\mathrm{q}}=d x_{1} \wedge d x_{2} \wedge \cdots \wedge d x_{\mathrm{q}}
$$

therefore using the Euclidean metric in place of $\tilde{g}$ yields

$$
c(g, \tilde{g})=1 .
$$

This simplifies the expression of (4.2), when use the Euclidean metric to compute the spherical measure of a submanifold.

Example 4.3 Using graded coordinates $x_{j}$ in a homogeneous group $\mathbb{G}$, we consider the standard Euclidean metric $\tilde{g}$ given by $\delta_{i j}$, along with our fixed left invariant Riemannian metric $g$. We notice that the volume measure $\operatorname{vol}_{\tilde{g}}$ associated to $\tilde{g}$ is left invariant. Let $\Sigma$ be a non-horizontal submanifold of codimension $k$, with unit normal $\tilde{\mathbf{n}}_{E}$ with repect to the Euclidean metric $\tilde{g}$. We define $k$-vector

$$
\tilde{\mathbf{n}}_{E, g}(p)=\left(g_{k}^{*}\right)^{-1} \tilde{g}_{k}^{*}\left(\tilde{\mathbf{n}}_{E}(p)\right),
$$


hence [19, Proposition 3.1] gives

$$
\left\|\tilde{\mathbf{n}}_{E, g, H}\right\|_{g}=\sqrt{\sum_{1 \leq j_{1}<j_{2}<\cdots<j_{k} \leq \mathrm{m}}\left\langle\tilde{\mathbf{n}}_{E}, X_{j_{1}} \wedge \cdots \wedge X_{j_{k}}\right\rangle_{E}^{2}}
$$

where $\langle\cdot, \cdot\rangle_{E}$ denotes the Euclidean scalar product on $k$-vectors and

$$
\tilde{\mathbf{n}}_{E, g, H}=\pi_{g, H}\left(\tilde{\mathbf{n}}_{E, g}\right) .
$$

Since in this case $c(g, \tilde{g})=1$, combining (4.1) and (4.2), we obtain

$$
\mathcal{S}_{d}^{\mathrm{Q}-k}(B)=\int_{B} \sqrt{\sum_{1 \leq j_{1}<j_{2}<\cdots<j_{k} \leq \mathrm{m}}\left\langle\tilde{\mathbf{n}}_{E}, X_{j_{1}} \wedge \cdots \wedge X_{j_{k}}\right\rangle_{E}^{2}} d \mathcal{H}_{E}^{\mathrm{q}-k}(p)
$$

for every Borel set $B \subset \Sigma$ and for any (q $-k$ )-vertically symmetric homogeneous distances $d$. The symbol $\mathcal{H}_{E}^{\mathrm{q}-k}$ denotes the $(\mathrm{q}-k)$-dimensional Hausdorff measure with respect to the Euclidean distance. In particular, since all smooth hypersurfaces are non-horizontal submanifolds, the previous formula for $k=1$ yields the well known formula for the spherical measure $\mathcal{S}^{\mathrm{Q}-1}$ of hypersurfaces

$$
\mathcal{S}_{d}^{\mathrm{Q}-1}(\Sigma)=\int_{\Sigma} \sqrt{\sum_{j=1}^{\mathrm{m}}\left\langle\tilde{\mathbf{n}}_{E}, X_{j}\right\rangle_{E}^{2}} d \mathcal{H}_{E}^{\mathrm{q}-1}(p)
$$

that is here extended to the largest class of suitably symmetric distances, namely the $(q-1)$-vertically symmetric distances. In this case $\tilde{n}_{E}$ is a normal to $\Sigma$ with respect to the Euclidean metric.

Corollary 4.4 (Coarea formula) Let $1 \leq k \leq \mathrm{m}$ and let $f: A \rightarrow \mathbb{R}^{k}$ be a Riemannian Lipschitz map, where $A \subset \mathbb{G}$ is measurable. We consider a homogeneous distance d on $\mathbb{G}$ that is $(\mathrm{q}-k)$-vertically symmetric. By Theorem 1.1, $d$ is rotationally symmetric with respect to $\mathcal{N}_{\mathrm{q}-k}$, hence we denote by $\omega_{d}\left(\mathcal{N}_{\mathrm{q}-k}\right)$ the associated constant spherical factor. Thus, defining the rescaled spherical measure

$$
\mathcal{S}_{d}^{\mathrm{Q}-k}=\omega_{d}\left(\mathcal{N}_{\mathrm{q}-k}\right) \mathcal{S}^{\mathrm{Q}-k}
$$

for any nonnegative measurable function $u: A \rightarrow \mathbb{R}$, we have

$$
\int_{A} u(x) J_{g, H} f(x) d \operatorname{vol}_{g}(x)=\int_{\mathbb{R}^{k}}\left(\int_{f^{-1}(t)} u(x) \mathcal{S}_{d}^{\mathrm{Q}-k}(x)\right) d t,
$$

where vol $_{g}$ is the Riemannian volume measure on $\mathbb{G}$ and

$$
J_{g, H}(x)=\left\|\pi_{x, k}\left(\nabla f_{1}(x) \wedge \nabla f_{2}(x) \wedge \cdots \wedge \nabla f_{k}(x)\right)\right\|
$$

is the horizontal Jacobian at every differentiability point $x \in A$ of $f$. 
We point out that the class of rotationally symmetric distances for which the coarea formula (4.5) holds is larger than the family of distances considered in [19]. The arguments to establish the previous corollary are the same ones of [19, Theorem 1.2]. In our case they are combined with the area formula (4.2). If we divide both members of (4.5) by $c(g, \tilde{g})$, we immediately establish that

$$
\int_{A} u(x) J_{g, H} f(x) d \operatorname{vol}_{\tilde{g}}(x)=\int_{\mathbb{R}^{k}}\left(\int_{f^{-1}(t)} u(x) \mathcal{S}_{d, \tilde{g}}^{\mathrm{Q}-k}(x)\right) d t,
$$

under the assumptions of Corollary 4.4. We have set $\mathcal{S}_{d, \tilde{g}}^{\mathrm{Q}-k}=\mathcal{S}_{d}^{\mathrm{Q}-k} / c(g, \tilde{g})$.

\section{Multiradial Distances}

In this section, we study integral formulas for the spherical measure with respect to a multiradial distance, that is another type of "rotational symmetric distance". Two important classes of multiradial distances are the Cygan-Korányi distance (Example 3.2) and the $d_{\infty}$ distance (Example 6.2).

Definition 5.1 (Multiradial distance) We say that a homogeneous distance $d$ on a homogeneous group $\mathbb{G}$ is multiradial if there exists $\varphi:[0,+\infty)^{\iota} \rightarrow[0,+\infty)$ continuous and monotone nondecreasing on each single variable, such that

$$
d(x, 0)=\varphi\left(\left|x_{1}\right|, \ldots,\left|x_{\iota}\right|\right),
$$

$x_{j}=P_{H^{j}}(x)$ and $P_{H^{j}}: \mathbb{G} \rightarrow H^{j}$ is the canonical projection with respect to the direct sum decomposition of $\mathbb{G}$ into subspaces $H^{j}$.

As an easy consequence of 1-homogeneity of $d$ with respect to dilations, one may also observe that $\varphi$ in the definition of multiradial distance satisfies

$$
\varphi(x) \rightarrow+\infty \text { as }|x| \rightarrow+\infty
$$

where $|\cdot|$ is the Euclidean norm of $\mathbb{R}^{\iota}$.

The following proposition shows that the assumptions of Theorem 1.1 also hold when the distance $d$ is multiradial.

Proposition 5.1 If $d: \mathbb{G} \times \mathbb{G} \rightarrow[0,+\infty)$ is multiradial, then it is also n-vertically symmetric for every $\mathrm{n}=1, \ldots, \mathrm{q}-1$.

Proof We represent the metric unit ball as follows

$$
\mathbb{B}(0,1)=\left\{x \in \mathbb{G}: \varphi\left(\left|x_{1}\right|, \ldots,\left|x_{l}\right|\right) \leq 1\right\},
$$

observing that in general it need not be convex. Let us fix $\ell=\ell_{\mathrm{n}}$, referring to (3.2). Let us consider the nontrivial case where

$$
\mathrm{n}=J+\mathrm{h}_{\ell+1}+\cdots+\mathrm{h}_{\iota}
$$


and $0<j<\mathrm{h}_{\ell}$, we consider two $j$-dimensional subspaces $S_{1}, S_{2} \subset H^{\ell}$. We choose any isometry $J: H^{\ell} \rightarrow H^{\ell}$ with $J\left(S_{1}\right)=S_{2}$ with respect to the fixed graded scalar product on $\mathbb{G}$ and set $\mathcal{F}$ to be the set of all isometries of $H^{\ell}$. The map $T: \mathbb{G} \rightarrow \mathbb{G}$ defined as

$$
\left.T\right|_{H^{\ell}}=J \text { and }\left.T\right|_{H^{j}}=\operatorname{Id}_{H^{j}}
$$

for all $j \neq \ell$, is clearly an isometry of $\mathbb{G}$. The special representation (5.2) of the metric unit ball clearly gives

$$
T(\mathbb{B}(0,1))=\mathbb{B}(0,1) .
$$

Let us consider the projection $P_{V}: \mathbb{G} \rightarrow V$ with $V=H^{1} \oplus \cdots \oplus H^{\ell}$ and observe that

$$
P_{V}(\mathbb{B}(0,1)) \subset \mathbb{B}(0,1) \cap V=\left\{x \in V: d(x, 0)=\varphi\left(\left|x_{1}\right|, \ldots,\left|x_{\ell}\right|, 0, \ldots, 0\right) \leq 1\right\}
$$

in view of the nondecreasing monotonicity of $\varphi$ with respect to each single variable. Clearly, any element $v \in \mathbb{B}(0,1) \cap V$ is equal to $P_{V}(v)$, therefore

$$
\mathbb{B}(0,1) \cap V \subset P_{V}(\mathbb{B}(0,1)) .
$$

If we set $\psi:\left(H^{1} \oplus \cdots \oplus H^{\ell-1}\right) \times[0,+\infty) \rightarrow[0,+\infty)$ as

$$
\psi(u, t)=\varphi\left(\left|P_{H^{1}} u\right|, \ldots,\left|P_{H^{\ell-1}} u\right|, t, 0, \ldots, 0\right),
$$

then also property (1) of Definition 3.2 is established, that is $d$ is an n-vertically symmetric distance.

Remark 5.2 As a consequence of Proposition 5.1 joined with Theorem 1.1, the area formulas (1.3) and (4.2), and the coarea formula (4.5) hold with respect to any multiradial distance.

\subsection{Multiradial Distances in Step Two Homogeneous Groups}

The next theorem shows that in step two homogeneous groups the spherical factor with respect to a multiradial distance has a simple formula. The same formula will be obtained in Sect. 6 for all homogeneous distances such that their metric unit ball is convex.

Theorem 5.3 If $\mathbb{G}$ is a step two homogeneous group and $d$ is a multiradial distance, then for every n-dimensional homogeneous subspace $V \subset \mathbb{G}$ we have

$$
\beta_{d}(V)=\mathcal{H}_{|\cdot|}^{\mathrm{n}}(\mathbb{B} \cap V)
$$

where $1 \leq \mathrm{n} \leq \mathrm{q}-1$ and $\mathbb{B}=\{x \in \mathbb{G}: d(x, 0) \leq 1\}$. 
Proof We choose $z \in \mathbb{B}$ and write $V=V_{1} \oplus V_{2}$ with $V_{j} \subset H^{j}$, being $V$ a homogeneous subspace of $\mathbb{G}$. Then the assumptions on $d$ ensure that $\mathbb{B}$ is defined as in (5.2), therefore we get

$$
V \cap \mathbb{B}(z, 1)=\left\{v \in V: \varphi\left(\left|P_{H^{1}}\left(z^{-1} v\right)\right|, \mid P_{H^{2}}\left(z^{-1} v \mid\right) \leq 1\right\}\right.
$$

The BCH formula (2.2) yields

$$
V \cap \mathbb{B}(z, 1)=\left\{v_{1}+v_{2} \in V: \varphi\left(\left|v_{1}-z_{1}\right|,\left|v_{2}-z_{2}-\frac{1}{2}\left[z_{1}, v_{1}\right]\right|\right) \leq 1\right\}
$$

with $z_{j}=P_{H^{j}}(z)$ and $v_{j}=P_{H^{j}}(v)$. From the coercivity of $\varphi$ we can define

$$
r_{1}=\sup \{t \geq 0: \varphi(t, 0) \leq 1\} \in(0,+\infty)
$$

then considering an orthogonal system of coordinates on $V$ and denoting by $\mathcal{L}^{\text {n }}$ the corresponding Lebesgue measure on $V$, Fubini's theorem yields

$$
\begin{aligned}
\mathcal{H}_{|\cdot|}^{\mathrm{n}}(V \cap \mathbb{B}(z, 1)) & =\mathcal{L}^{\mathrm{n}}(V \cap \mathbb{B}(z, 1)) \\
& =\int_{V_{1} \cap B_{E}\left(z_{1}, r_{1}\right)} \mathcal{L}^{\mathrm{n}_{2}}\left(\left\{v_{2} \in V_{2}: v_{1}+v_{2} \in \mathbb{B}(z, 1)\right\}\right) d v_{1}
\end{aligned}
$$

where $B_{E}(z, r)=\{x \in \mathbb{G}:|x-z|<r\}$ and $\mathrm{n}_{j}=\operatorname{dim} V_{j}$ with $j=1,2$. From the nondecreasing monotonicity of $\varphi$ with respect to each variable, defining

$$
\rho(x)=\sup \{t \geq 0: \varphi(|x|, t) \leq 1\}
$$

whenever $|x|<r_{1}$ gives the monotonicity

$$
\rho\left(w_{2}\right) \leq \rho\left(w_{1}\right) \quad \text { for } \quad\left|w_{1}\right| \leq\left|w_{2}\right|<r_{1}
$$

As a consequence, using the integral representation (5.4) for $z=0$ we get the formula

$$
\begin{aligned}
\mathcal{H}_{|\cdot|}^{\mathrm{n}}(V \cap \mathbb{B}(0,1)) & =\int_{V_{1} \cap B_{E}\left(0, r_{1}\right)} \mathcal{L}^{\mathrm{n}_{2}}\left(\left\{v_{2} \in V_{2}: \varphi\left(\left|v_{1}\right|,\left|v_{2}\right|\right) \leq 1\right\}\right) d v_{1} \\
& =\int_{V_{1} \cap B_{E}\left(0, r_{1}\right)} \mathcal{L}^{\mathrm{n}_{2}}\left(V_{2} \cap B_{E}\left(0, \rho\left(v_{1}\right)\right)\right) d v_{1},
\end{aligned}
$$

that will be used later. Defining the function $\Psi\left(z, v_{1}\right)=z_{2}+\frac{1}{2}\left[z_{1}, v_{1}\right]$, we have

$$
\begin{aligned}
\mathcal{H}_{|\cdot|}^{\mathrm{n}}(V \cap \mathbb{B}(z, 1)) & =\int_{V_{1} \cap B_{E}\left(z_{1}, r_{1}\right)} \mathcal{L}^{\mathrm{n}_{2}}\left(V_{2} \cap B_{E}\left(\Psi\left(z, v_{1}\right), \rho\left(v_{1}-z_{1}\right)\right)\right) d v_{1} \\
& =\int_{\left(V_{1}-z_{1}\right) \cap B_{E}\left(0, r_{1}\right)} \mathcal{L}^{\mathrm{n}_{2}}\left(V_{2} \cap B_{E}\left(\Psi\left(z, v_{1}\right), \rho\left(v_{1}\right)\right)\right) d v_{1}
\end{aligned}
$$




$$
\leq \int_{V_{1} \cap B_{E}\left(0, r_{1}\right)} \mathcal{L}^{\mathrm{n}_{2}}\left(\left(V_{2}-\Psi\left(z, v_{1}\right)\right) \cap B_{E}\left(0, \rho\left(v_{1}\right)\right)\right) d v_{1}
$$

Taking into account the last inequality, Theorem 6.3 and formula (5.6), we get

$$
\mathcal{H}_{|\cdot|}^{\mathrm{n}}(V \cap \mathbb{B}(z, 1)) \leq \int_{V_{1} \cap B_{E}\left(0, r_{1}\right)} \mathcal{L}^{\mathrm{n}_{2}}\left(V_{2} \cap B_{E}\left(0, \rho\left(v_{1}\right)\right)\right) d v_{1}=\mathcal{H}_{|\cdot|}^{\mathrm{n}}(V \cap \mathbb{B}),
$$

therefore concluding the proof.

By (5.3) we show that a multiradial distance is also rotationally symmetric with respect to "large families" of homogeneous subspaces.

Theorem 5.4 Let $\mathbb{G}$ be a homogeneous group of step two and let $d$ be a multiradial distance. Let $1 \leq \mathrm{n}_{1}, \mathrm{n}_{2} \leq \mathrm{q}-1$ be two integers such that $\mathrm{n}_{1}+\mathrm{n}_{2}=\mathrm{n} \leq \mathrm{q}-1$ and let $\mathcal{F}_{\mathrm{n}_{1}, \mathrm{n}_{2}}$ be the family of all homogeneous subspaces $V=V_{1} \oplus V_{2} \subset \mathbb{G}$ such that $\operatorname{dim} V_{1}=\mathrm{n}_{1}$ and $\operatorname{dim} V_{2}=\mathrm{n}_{2}$. Then $d$ is rotationally symmetric with respect to $\mathcal{F}_{\mathrm{n}_{1}, \mathrm{n}_{2}}$.

Proof Since $\mathbb{G}$ is of step two, we have $\mathbb{G}=H^{1} \oplus H^{2}$. Let us consider two homogeneous subspaces $V$ and $W$ with direct decompositions $V_{1} \oplus V_{2}$ and $W_{1} \oplus W_{2}$, respectively, where $V_{1}, W_{1} \subset H^{1}, V_{2}, W_{2} \subset H^{2}$ and we have the conditions

$$
\operatorname{dim} V_{1}=\operatorname{dim} W_{1}=\mathrm{n}_{1} \text { and } \operatorname{dim} V_{2}=\operatorname{dim} W_{2}=\mathrm{n}_{2} .
$$

Considering two isometries $J_{1}: H^{1} \rightarrow H^{1}$ and $J_{2}: H^{2} \rightarrow H^{2}$ such that

$$
J_{1}\left(V_{1}\right)=W_{1} \text { and } J_{2}\left(V_{2}\right)=W_{2},
$$

respectively, then for every $x_{1} \in H^{1}$ and $x_{2} \in H^{2}$ we define

$$
T\left(x_{1}+x_{2}\right)=J_{1}\left(x_{1}\right)+J_{2}\left(x_{2}\right) .
$$

The mapping $T: \mathbb{G} \rightarrow \mathbb{G}$ is an isometry, since $H^{1}$ is orthogonal to $H^{2}$ with respect to our fixed graded scalar product on $\mathbb{G}$. Moreover, the shape of the metric unit ball $\mathbb{B}$ gives the equalities

$$
\begin{aligned}
T(\mathbb{B} \cap V) & =T\left(\left\{x_{1}+x_{2} \in \mathbb{G}: x_{1} \in V_{1}, x_{2} \in V_{2}, \varphi\left(\left|x_{1}\right|,\left|x_{2}\right|\right) \leq 1\right\}\right) \\
& =\left\{T\left(x_{1}\right)+T\left(x_{2}\right) \in \mathbb{G}: x_{1} \in V_{1}, x_{2} \in V_{2}, \varphi\left(\left|x_{1}\right|,\left|x_{2}\right|\right) \leq 1\right\} \\
& =\left\{T\left(x_{1}\right)+T\left(x_{2}\right) \in \mathbb{G}: x_{1} \in V_{1}, x_{2} \in V_{2}, \varphi\left(\left|T\left(x_{1}\right)\right|,\left|T\left(x_{2}\right)\right|\right) \leq 1\right\} \\
& =\left\{y_{1}+y_{2} \in \mathbb{G}: y_{1} \in W_{1}, y_{2} \in W_{2}, \varphi\left(\left|y_{1}\right|,\left|y_{2}\right|\right) \leq 1\right\} \\
& =\mathbb{B} \cap W .
\end{aligned}
$$

As a consequence, formula (5.3) concludes the proof. 


\subsection{One Dimensional Rotational Symmetries}

We show that any multiradial distance has rotational symmetries with respect to one dimensional homogeneous subspaces of the Lie algebra.

Theorem 5.5 Let $d$ be a multiradial distance on a homogeneous group $\mathbb{G}$ and let $1 \leq \mathrm{N} \leq \iota$. Then for every one dimensional subspace $L \subset H^{\mathrm{N}}$ we have

$$
\beta_{d}(L)=\mathcal{H}_{|\cdot|}^{1}(\mathbb{B} \cap L),
$$

where $\mathbb{B}=\{x \in \mathbb{G}: d(x, 0) \leq 1\}$.

Proof We consider a one dimensional subspace $L$ of $H^{\mathrm{N}}$. We observe that

$$
L \cap \mathbb{B}(z, 1)=\left\{v \in L: z^{-1} v \in \mathbb{B}\right\}
$$

Since $d$ is multiradial, we may write

$$
L \cap \mathbb{B}(z, 1)=\left\{v \in L: \varphi\left(\left|P_{H^{1}}\left(z^{-1} v\right)\right|, \ldots, \mid P_{H^{l}}\left(z^{-1} v \mid\right) \leq 1\right\} .\right.
$$

The nondecreasing monotonicity of $\varphi$ with respect to each variable shows that

$$
L \cap \mathbb{B}(z, 1) \subset\left\{v \in L: \varphi(\underbrace{0, \ldots, 0}_{\mathrm{N}-1 \text { zeros }},\left|v-P_{H^{\mathrm{N}}}(z)\right|, 0, \ldots, 0) \leq 1\right\}=\zeta_{z}+J_{L},
$$

where $\zeta_{z}=P_{H^{\mathrm{N}}}(z)$ and $J_{L}=\{v \in L: \varphi(0, \ldots, 0,|v|, 0, \ldots, 0) \leq 1\}$. The previous inclusion yields

$$
\mathcal{H}_{|\cdot|}^{1}(L \cap \mathbb{B}(z, 1)) \leq \mathcal{H}_{|\cdot|}^{1}\left(\zeta_{z}+J_{L}\right)=\mathcal{L}^{1}\left(J_{L}\right)
$$

Observing that

$$
\mathcal{H}_{|\cdot|}^{1}(L \cap \mathbb{B})=\mathcal{H}_{|\cdot|}^{1}(\{v \in L: \varphi(0, \ldots, 0,|v|, 0, \ldots, 0) \leq 1\})=\mathcal{L}^{1}\left(J_{L}\right)
$$

we have shown our claim.

Theorem 5.6 Let $d$ be a multiradial distance on a homogeneous group $\mathbb{G}$ and let $1 \leq \mathrm{N} \leq \iota$. Then $d$ is rotationally symmetric with respect to the family of all one dimensional subspaces of $H^{\mathrm{N}} \subset \mathbb{G}$.

Proof Let us consider the one dimensional subspaces $V, W \subset H^{\mathrm{N}}$ and define the isometries $J_{i}: H^{i} \rightarrow H^{i}$ such that

$$
J_{\mathrm{N}}(V)=W \quad \text { and } \quad J_{i}=\operatorname{Id}_{H^{i}},
$$


for all $i \neq \mathrm{N}$ with $1 \leq i \leq \iota$. For $x_{i} \in H^{i}$, we define

$$
T\left(x_{1}+\cdots+x_{\iota}\right)=J_{1}\left(x_{1}\right)+J_{2}\left(x_{2}\right)+\cdots+J_{\iota}\left(x_{\iota}\right)
$$

so observing that the subspaces $H^{i}$ are orthogonal we conclude that $T: \mathbb{G} \rightarrow \mathbb{G}$ is an isometry. The fact that $d$ is multiradial implies that

$$
\begin{aligned}
T(\mathbb{B} \cap V) & =T\left(\left\{x \in H^{\mathrm{N}}: x \in V, \varphi(\underbrace{0, \ldots, 0}_{\mathrm{N}-1 \text { zeros }},|x|, 0, \ldots) \leq 1\right\}\right) \\
& =\left\{J_{\mathrm{N}}(x) \in H^{\mathrm{N}}: x \in V, \varphi(0, \ldots,|x|, 0, \ldots) \leq 1\right\} \\
& =\{y \in W: \varphi(0, \ldots,|y|, 0, \ldots) \leq 1\} \\
& =\mathbb{B} \cap W .
\end{aligned}
$$

As a consequence, formula (5.7) concludes the proof.

Remark 5.7 Let us point out that all one dimensional subspaces of a homogeneous group are automatically Lie subgroups.

\subsection{Proof of Theorem 1.3 and Applications}

In this section we apply the previous results to obtain a standard form of area formulas with respect to multiradial distances. Throughout this section, $\mathbb{G}$ is a fixed homogeneous group and $\Sigma \subset \mathbb{G}$ is an n-dimensional $C^{1}$ smooth submanifold of degree N.

Proof of Theorem 1.3 Let us now assume that conditions (1) hold. If $V \in \mathcal{F}$ is any homogeneous subgroup of the form $V=V_{1} \oplus V_{2}$, with $\operatorname{dim} V_{1}=\mathrm{n}_{1}$ and $\operatorname{dim} V_{2}=\mathrm{n}_{2}$, then we must have

$$
\mathrm{n}_{1}+\mathrm{n}_{2}=\mathrm{n} \text { and } \mathrm{n}_{1}+2 \mathrm{n}_{2}=\mathrm{N} \text {. }
$$

The previous conditions uniquely define the integers

$$
\mathrm{n}_{1}=2 \mathrm{n}-\mathrm{N} \text { and } \mathrm{n}_{2}=\mathrm{N}-\mathrm{n} .
$$

By virtue of Theorem 5.4, $d$ is rotationally symmetric with respect to $\mathcal{F}$ and $\omega_{d}(\mathcal{F})$ denotes its constant spherical factor. By our assumptions the homogeneous tangent space at points of maximum degree $\mathrm{N}$ is a homogeneous subgroup. Due to [23, Proposition 3.3] and [23, Proposition 3.5], its topological dimension is $\mathrm{n}$ and its Hausdorff dimension is N. We are also in the assumptions of [23, Theorem 1.3], so the area formula (1.7) of [23] exactly gives (1.4).

Let us assume now that conditions (2) hold, namely $\Sigma$ is one dimensional. Again from [23, Proposition 3.3] and [23, Proposition 3.5], at points of maximum degree the homogeneous tangent space is a one dimensional subspace of $H^{\mathrm{N}}$, that is automatically a one dimensional homogeneous subgroup of Hausdorff dimension N. By 
Theorem 5.6 the distance $d$ is rotationally symmetric with respect to the family $\mathcal{F}$ of all one dimensional homogeneous subgroups of Hausdorff dimension N. Applying the area formula (1.7) of [23] we immediately establish (1.4), concluding the proof.

A special application of the previous theorem is the following.

Corollary 5.8 If $\Sigma$ is a $C^{1,1}$ smooth submanifold in a step two homogeneous group $\mathbb{G}$ equipped with a multiradial distance $d$, then there exists a constant $\omega_{d}(\mathcal{F})$ as in Theorem 1.3 such that (1.4) holds, with $\mathcal{S}_{d}^{\mathrm{N}}=\omega_{d}(\mathcal{F}) \mathcal{S}^{\mathrm{N}}$.

Proof Due to the $C^{1,1}$ smoothness, we may apply [20, Corollary 1.2], concluding that the subset of $\Sigma$ made by all points of degree less than $\mathrm{N}$ is $\mathcal{S}^{\mathrm{N}}$-negligible. The same smoothness, by [26, Lemma 3.9], implies that the homogeneous tangent space at all points of maximum degree is a homogeneous subgroup. We are precisely in the assumptions (1) of Theorem 1.3, hence (1.4) is established.

A simple application of the previous corollary is given in the next example.

Example 5.9 Let us consider an open, bounded and connected set $U \subset \mathbb{R}^{\mathrm{n}}$, assuming that there exists another open set $\tilde{U}$ with $\bar{U} \subset \tilde{U}$ and a smooth embedding $\Phi: \tilde{U} \rightarrow \mathbb{G}$ of class $C^{1,1}$. We assume that $\mathbb{G}$ is equipped with a structure of $\mathrm{H}$-type group equipped with a multiradial distance that satisfies

$$
d(x, 0)=\sqrt[4]{\left|x_{1}\right|^{4}+16\left|x_{2}\right|^{2}}
$$

for all $x \in \mathbb{G}$, see Example 3.2. Let $|\cdot|$ be the norm on $\mathbb{G}$ associated to this H-type structure. We consider the $C^{1,1}$ submanifold $\Sigma=\Phi(U)$ and denote its degree by $\mathrm{N}$. Therefore we have two uniquely defined integers

$$
n_{1}=2 n-\mathrm{N} \text { and } n_{2}=\mathrm{N}-n
$$

that satisfy $\mathrm{n}=\mathrm{n}_{1}+\mathrm{n}_{2}$ and $\mathrm{N}=\mathrm{n}_{1}+2 \mathrm{n}_{2}$. By Theorem 5.4, $d$ is rotationally invariant with respect to the family $\mathcal{F}_{2, \mathbb{G}}$ of all n-dimensional homogeneous subgroups $V$ of Hausdorff dimension $\mathrm{N}$. We denote by $\omega_{d}\left(\mathcal{F}_{2, \mathbb{G}}\right)$ the associated constant spherical factor and introduce the renormalized spherical measure $\mathcal{S}_{d}^{\mathrm{N}}=\omega_{d}\left(\mathcal{F}_{2, \mathbb{G}}\right) \mathcal{S}^{\mathrm{N}}$. We denote by $y=\left(y_{1}, \ldots, y_{\mathrm{n}}\right)$ the local coordinates of $\Sigma$ with respect to $\Phi$. As a consequence of Corollary 5.8 and [23, Proposition 7.5], we get

$$
\mathcal{S}_{d}^{\mathrm{N}}(\Sigma)=\int_{U}\left\|\pi_{\Phi(y), \mathrm{N}}\left(\partial_{y_{1}} \Phi(y) \wedge \cdots \wedge \partial_{y_{\mathrm{n}}} \Phi(y)\right)\right\|_{g} d y,
$$

where $g$ is the left invariant Riemannian metric associated to fixed norm $|\cdot|$ on $\mathbb{G}$.

Remark 5.10 Assuming that we have a transversal $C^{1}$ smooth curve $\Sigma$ in the Heisenberg group $\mathbb{H}^{n}$, namely a non-horizontal curve, then Theorem 1.2 yields the area formula

$$
\mathcal{S}_{d}^{2}\left\llcorner\Sigma(B)=\int_{B}\left\|\tau_{\Sigma, \mathrm{N}}^{\tilde{g}}(p)\right\|_{g} d \sigma_{\tilde{g}}(p)\right.
$$


for any homogeneous distance. We do not need further assumptions on the distance. From Definition 3.2 one may easily notice that in $\mathbb{H}^{n}$ any homogeneous distance is automatically 1-vertically symmetric. In particular, formula (5.9) holds for the subRiemannian distance of $\mathbb{H}^{n}$, that is not multiradial.

\section{Sections of Convex Balls}

In this section, we study the spherical factor of those homogeneous distances whose metric unit ball is a convex set. Such distances can be found in any homogeneous group, according to the next examples.

Example 6.1 Theorem 2 of [13] shows that in a homogeneous group we can find a homogeneous distance whose metric unit ball is an Euclidean ball with suitably small radius, where all layers $H^{j}$ in the decomposition of $\mathbb{G}$ are orthogonal. In particular, the metric unit ball is a convex set.

Example 6.2 The homogeneous distance $d_{\infty}$ of [10] can be easily checked to have a convex metric unit ball. This distance is also multiradial.

The Cygan-Korányi distance of Example 3.2 has a convex metric unit ball and it is multiradial. The following fact is our crucial tool.

Theorem 6.3 Let $H$ be a q-dimensional Hilbert space with $\mathrm{q} \geq 2$ and let $C \subset H$ be a compact and convex set, whose interior is nonempty and it contains the origin. Let $S$ denote an $\mathrm{n}$-dimensional subspace of $H$ and consider $V=S^{\perp}$ its orthogonal subspace. Then the subset $D=\{v \in V: C \cap(v+S) \neq \emptyset\}$ is convex and $\psi: D \rightarrow$ $[0,+\infty)$, defined as follows

$$
\psi(v)=\left[\mathcal{H}_{|\cdot|}^{\mathrm{n}}(C \cap(v+S))\right]^{1 / \mathrm{n}}
$$

is concave on $D$.

Proof It is easy to observe that $D$ is convex and with nonempty interior in $V$. Let us consider $v, w \in D$ and $\theta \in(0,1)$. Convexity of $C$ gives

$$
\theta((v+S) \cap C)+(1-\theta)((w+S) \cap C) \subset([\theta v+(1-\theta) w]+S) \cap C,
$$

that it can be rewritten as follows 


$$
((\theta v+S) \cap \theta C)+(((1-\theta) w+S)) \cap(1-\theta) C) \subset([\theta v+(1-\theta) w]+S) \cap C .
$$

Our point is to use the the classical Brunn-Minkowski inequality, in dimension $\mathrm{n}$. We observe the following equality of sets

$$
(\gamma u+S) \cap \gamma C=\gamma u+(S \cap(\gamma C-\gamma u))
$$

for every $\gamma \in \mathbb{R}$ and $u \in H$, hence we define

$$
C(\gamma, u)=S \cap(\gamma C-\gamma u) .
$$

Thus, the inclusion (6.1) can be written as follows

$$
[\theta v+C(\theta, v)]+[(1-\theta) w+C((1-\theta), w)] \subset([\theta v+(1-\theta) w]+S) \cap C
$$

It follows that

$$
\theta v+(1-\theta) w+C(\theta, v)+C((1-\theta), w) \subset([\theta v+(1-\theta) w]+S) \cap C .
$$

The Brunn-Minkowski inequality in $S$ gives

$$
\left[\mathcal{H}_{|\cdot|}^{\mathrm{n}}(C(\theta, v)+C((1-\theta), w))\right]^{1 / \mathrm{n}} \geq\left[\mathcal{H}_{|\cdot|}^{\mathrm{n}}(C(\theta, v))\right]^{1 / \mathrm{n}}+\left[\mathcal{H}_{|\cdot|}^{\mathrm{n}}(C((1-\theta), w))\right]^{1 / \mathrm{n}}
$$

Taking into account that

$$
C(\theta, u)=\theta C(1, u) \text { and } C((1-\theta), u)=(1-\theta) C(1, u) \text {, }
$$

the inequality (6.4) joined with the inclusion (6.3), we obtain

$$
\psi(\theta v+(1-\theta) w) \geq \theta\left[\mathcal{H}_{|\cdot|}^{\mathrm{n}}(C(1, v))\right]^{1 / \mathrm{n}}+(1-\theta)\left[\mathcal{H}_{|\cdot|}^{\mathrm{n}}(C(1, w))\right]^{1 / \mathrm{n}}
$$

Finally, we observe that

$$
\psi(u)=\left[\mathcal{H}_{|\cdot|}^{\mathrm{n}}(C(1, u))\right]^{1 / \mathrm{n}}
$$

for each $u \in D$, hence completing the proof.

We are now in the position to prove an explicit formula for the spherical factor when the metric unit ball is convex. 
Proof of Theorem 1.4 According to the definition of vertical subgroup, we set

$$
N=N_{\ell} \oplus H^{\ell+1} \oplus \cdots \oplus H^{\iota}
$$

for $\ell=\ell_{\mathrm{n}}$, where $\ell_{\mathrm{n}}$ is defined in (3.2). Applying Lemma 3.5, we get

$$
\mathcal{H}_{|\cdot|}^{\mathrm{n}}(N \cap \mathbb{B}(z, 1))=\mathcal{H}_{|\cdot|}^{\mathrm{n}}\left(\mathbb{B}(0,1) \cap z^{-1} N\right) .
$$

To study the previous function with respect to $z$, we define

$$
a(z)=\mathcal{H}_{|\cdot|}^{\mathrm{n}}(\mathbb{B}(0,1) \cap z N)
$$

We set $V=N^{\perp}$, that can be written as follows

$$
V=H^{1} \oplus \cdots \oplus H^{\ell-1}+S_{\ell}
$$

This is a homogeneous subspace of $\mathbb{G}$, that need not be a subgroup. However Lemma 3.3 gives the mappings

$$
P_{V}: \mathbb{G} \rightarrow V \text { and } \Pi_{N}: \mathbb{G} \rightarrow N,
$$

such that for $y \in \mathbb{G}$ we have

$$
y=P_{V}(y) \Pi_{N}(y),
$$

where $P_{V}$ is the projection onto $V$ with respect to the direct sum $\mathbb{G}=V \oplus N$. As a consequence, we can write

$$
a(z)=\mathcal{H}_{|\cdot|}^{\mathrm{n}}(\mathbb{B}(0,1) \cap z N)=\mathcal{H}_{|\cdot|}^{\mathrm{n}}\left(\mathbb{B}(0,1) \cap P_{V}(z) N\right) .
$$

We are in the assumptions to apply (3.9), hence $P_{V}(z) N=P_{V}(z)+N$. Our special representation of $\mathbb{G}$ also allows us to have

$$
\mathbb{B}(0,1)^{-1}=-\mathbb{B}(0,1)
$$

It follows that

$$
a(z)=\mathcal{H}_{|\cdot|}^{\mathrm{n}}\left(\mathbb{B}(0,1) \cap\left(P_{V}(z)+N\right)\right)=\mathcal{H}_{|\cdot|}^{\mathrm{n}}\left(\mathbb{B}(0,1) \cap\left(-P_{V}(z)+N\right)\right)
$$

and the property $-P_{V}(z)=P_{V}\left(z^{-1}\right)$ yields

$$
a(z)=\mathcal{H}_{|\cdot|}^{\mathrm{n}}\left(\mathbb{B}(0,1) \cap\left(P_{V}\left(z^{-1}\right)+N\right)\right)=\mathcal{H}_{|\cdot|}^{\mathrm{n}}\left(\mathbb{B}(0,1) \cap\left(P_{V}\left(z^{-1}\right) N\right)\right) .
$$

Thus, by (6.6) we have proved that

$$
a(z)=\mathcal{H}_{|\cdot|}^{\mathrm{n}}\left(\mathbb{B}(0,1) \cap\left(z^{-1} N\right)\right)=a\left(z^{-1}\right)=a(-z),
$$


hence $a$ is even. We define $v=P_{V}\left(z^{-1}\right)$ and we introduce the even function

$$
b(t)=\left[\mathcal{H}_{|\cdot|}^{\mathrm{n}}(\mathbb{B}(0,1) \cap(t v+N))\right]^{1 / \mathrm{n}}
$$

for all $t \in \mathbb{R}$, observing that

$$
b(1)=\sqrt[\mathrm{n}]{a(z)} \text { and } b(0)=\sqrt[n]{\mathcal{H}_{|\cdot|}^{\mathrm{n}}(N \cap \mathbb{B}(0,1))} .
$$

Due to Theorem 6.3, the function $t \rightarrow b(t)$ is also concave on the compact interval

$$
I=\{t \in \mathbb{R}: \mathbb{B}(0,1) \cap(t v+N) \neq \emptyset\} .
$$

By (6.7) one easily observes that $I$ is an even interval, therefore $b: I \rightarrow[0,+\infty)$ takes its maximum at 0 , getting in particular that

$$
a(z) \leq \mathcal{H}_{|\cdot|}^{\mathrm{n}}(N \cap \mathbb{B}(0,1))
$$

Taking into account (6.5) and (6.8), we have proved that

$$
\beta_{d}(N)=\max _{z \in \mathbb{B}(0,1)} \mathcal{H}_{|\cdot|}^{\mathrm{n}}\left((N \cap \mathbb{B}(z, 1))=\mathcal{H}_{|\cdot|}^{\mathrm{n}}(N \cap \mathbb{B}(0,1)),\right.
$$

concluding the proof.

Open Access This article is licensed under a Creative Commons Attribution 4.0 International License, which permits use, sharing, adaptation, distribution and reproduction in any medium or format, as long as you give appropriate credit to the original author(s) and the source, provide a link to the Creative Commons licence, and indicate if changes were made. The images or other third party material in this article are included in the article's Creative Commons licence, unless indicated otherwise in a credit line to the material. If material is not included in the article's Creative Commons licence and your intended use is not permitted by statutory regulation or exceeds the permitted use, you will need to obtain permission directly from the copyright holder. To view a copy of this licence, visit http://creativecommons.org/licenses/by/4.0/.

\section{References}

1. Ambrosio, L., Ghezzi, R., Magnani, V.: BV functions and sets of finite perimeter in sub-Riemannian manifolds. Ann. Inst. H. Poincaré Anal. Non Linéaire 32(3):489-517, (2015)

2. Antonelli, G., Donato, D.D., Don, S., Donne, E.L.: Characterizations of uniformly differentiable cohorizontal intrinsic graphs in Carnot groups. Ann. Inst. Fourier (accepted)

3. Bellettini, G., Paolini, M., Venturini, S.: Some results on surface measures in calculus of variations. Ann. Mat. Pura Appl. 4(170), 329-357 (1996)

4. Capogna, L., Danielli, D., Garofalo, N.: The geometric Sobolev embedding for vector fields and the isoperimetric inequality. Commun. Anal. Geom. 2(2), 203-215 (1994)

5. Corni, F., Magnani, V.: Area formula for regular submanifolds of low codimension in Heisenberg groups. Adv. Calc. Var. (accepted)

6. Cygan, J.: Subadditivity of homogeneous norms on certain nilpotent Lie groups. Proc. Am. Math. Soc. 83(1), 69-70 (1981) 
7. Federer, H.: Geometric Measure Theory. Springer, New York (1969)

8. Folland, G.B., Stein, E.M.: Hardy Spaces on Homogeneous Groups. Princeton University Press, Princeton (1982)

9. Franchi, B., Serapioni, R.P., Serra Cassano, F.: Area formula for centered Hausdorff measures in metric spaces. Nonlinear Anal. 126, 218-233 (2015)

10. Franchi, B., Serapioni, R., Serra Cassano, F.: On the structure of finite perimeter sets in step 2 Carnot groups. J. Geom. Anal. 13(3), 421-466 (2003)

11. Franchi, B., Serapioni, R., Serra Cassano, F.: Regular submanifolds, graphs and area formula in Heisenberg groups. Adv. Math. 211(1), 152-203 (2007)

12. Garofalo, N., Nhieu, D.-M.: Isoperimetric and Sobolev inequalities for Carnot-Carathéodory spaces and the existence of minimal surfaces. Commun. Pure Appl. Math. 49(10), 1081-1144 (1996)

13. Hebisch, W., Sikora, A.: A smooth subadditive homogeneous norm on a homogeneous group. Studia Math. 96(3), 231-236 (1990)

14. Heinonen, J.: Lectures on Analysis on Metric Spaces. Universitext. Springer, New York (2001)

15. Julia, A., Golo, S.N., Vittone, D.: Area of intrinsic graphs and coarea formula in Carnot groups. arXiv:2004.02520 (2020)

16. Kirchheim, B., Serra Cassano, F.: Rectifiability and parameterization of intrinsic regular surfaces in the Heisenberg group. Ann. Sc. Norm. Super. Pisa Cl. Sci. (5), 3(4):871-896, 2004

17. Korte, R., Magnani, V.: Measure of curves in graded groups. Illinois J. Math. 56(2), 353-366 (2012)

18. Magnani, V.: Characteristic points, rectifiability and perimeter measure on stratified groups. J. Eur. Math. Soc. 8(4), 585-609 (2006)

19. Magnani, V.: Non-horizontal submanifolds and coarea formula. J. Anal. Math. 106, 95-127 (2008)

20. Magnani, V.: Blow-up estimates at horizontal points and applications. J. Geom. Anal. 20(3), 705-722 (2010)

21. Magnani, V.: On a measure theoretic area formula. Proc. R. Soc. Edinburgh A 145, 885-891 (2015)

22. Magnani, V.: A new differentiation, shape of the unit ball, and perimeter measure. Indiana Univ. Math. J. 66(1), 183-204 (2017)

23. Magnani, V.: Towards a theory of area in homogeneous groups. Calc. Var. Partial Differ. Equ. 58(3), 91 (2019)

24. Magnani, V.: Towards a theory of area in homogeneous groups. arXiv:1810.08094 (2018)

25. Magnani, V., Tyson, J.T., Vittone, D.: On transversal submanifolds and their measure. J. Anal. Math. 125(1), 319-351 (2015)

26. Magnani, V., Vittone, D.: An intrinsic measure for submanifolds in stratified groups. J. Reine Angew. Math. 619, 203-232 (2008)

27. Merlo, A.: Marstrand-Mattila rectifiability criterion for 1-codimensional measures in Carnot groups. arXiv:2007.03236 (2020)

28. Monti, R., Serra Cassano, F.: Surface measures in Carnot-Carathéodory spaces. Calc. Var. Partial Differ. Equ. 13(3), 339-376 (2001)

29. Pansu, P.: Geometrie du group d'heisenberg. These pour le titre de Doctour 3éme cycle, Université Paris VII (1982)

30. Varadarajan, V.S.: Lie Groups, Lie Algebras, and Their Representations, volume 102 of Graduate Texts in Mathematics. Springer, New York (1984). Reprint of the 1974 edition

31. Vittone, D.: Lipschitz graphs and currents in Heisenberg groups. arXiv:2007.14286 (2020)

Publisher's Note Springer Nature remains neutral with regard to jurisdictional claims in published maps and institutional affiliations. 